\title{
Histoire et état actuel de l'analyse des contraintes par rayons $\mathbf{X}$
}

\author{
B. Eigenmann et E. Macherauch \\ Institut für Werkstoffkunde 1, Universität Karlsruhe (TH), Germany
}

X-ray stress analyses on crystalline or partially crystalline materials are based on the determination of elastic lattice strains from the appertaining shift of diffraction profiles by means of monochromatic X-rays. Then, the lattice strains are converted to stresses using theory of elasticity. The practical as well as the scientific importance of X-ray stress analysis have been increased enormously during the passed three decades. Today, it is a widely spread tool for materials science and materials engineering. This is even more remarkable since during the 1950s the state of development and practical acceptance of X-ray stress analysis did not justify optimism concerning future perspectives. The pinciples of the method were difficult to understand and the necessary experimental efforts seemed to be too high for technical application. The evaluation procedures were not free of individual personal influences, and doubts arised concerning the meaning and the assessment of the measured stress values.

From the beginning of the $1960 \mathrm{~s}$, however, the situation was completely changed by the development of the $\sin ^{2} \psi$-method for X-ray stress analysis, by the development of experimental methods for the determination of precise X-ray elastic constants, and by the substitution of the classical X-ray film chambers for the registration of the interference lines by diffractometers usingproportional counters.

Since that time, many technical problems were investigated using X-ray stress analysis, and today it is worldwide the most widely applied method for residual stress determinations in several fields of research, engineering and quality control. Today, X-ray stress analyses are mainly performed using fully computer controlled four circle diffractometers and software packages allowing user friendly measurements and evaluations. However, the increasing knowledge about basic principles and difficulties in X-ray stress analysis opened a wide range of specific problems. Also the rapid development ofmaterials science and materials technology concerning, e.g., single crystalline materials, ceramics, and coatings requires a large variety ofnew specific measuring and evaluation methods. Consequently, in the field of $\mathrm{X}$-ray residual stress analysis, again a complex situation developed which is difficult to handle for many users in industry as well as in research. This paper sketches the historical development of X-ray stress analysis and gives a structured description of the actual state of the art. 


\section{INTRODUCTION}

L'analyse des contraintes par diffraction des rayons $\mathrm{X}$ sur des matériaux cristallins ou semicristallins est basée sur la détermination des déformations élastiques du réseau cristallins, auxquelles on associe des contraintes à l'aide de la théorie de l'élasticité. L'analyse des contraintes par rayons $\mathrm{X}$ a pris racine dans les années vingt, mais n'a valorisé dans la pratique que dans les trente-cinq dernières années. Elle représente aujourd'hui pour la science des matériaux et de l'ingénieur un domaine de travail aussi intéressant qu'important. Ceci est d'autant plus remarquable qu'elle se trouvait au milieu des années cinquante à un stade de développement qui laissait peu de place à l'optimisme. Les principes de cette méthode restaient alors troubles pour des utilisateurs surtout attachés à la pratique. Les montages servant à cette analyse étaient particulièrement difficiles, les résultats étaient entachés de subjectivité, et leur exploitation restait incertaine.

Le développement du procédé en $\sin ^{2} \psi$ de l'analyse des contraintes par rayons $\mathrm{X}$ et des méthodes donnant précisément les constantes élastiques radiocristallographiques (CER) ainsi que le remplacement des procédés classiques utilisant des films par des diffractomètres à compteurs proportionnels aboutirent au début des années soixante à des progrès remarquables de l'analyse des contraintes par rayons $\mathrm{X}$ en résolvant de nombreux problèmes théoriques et appliqués. Cette méthode d'analyse est aujourd'hui la méthode mondialement utilisée pour la détermination des contraintes résiduelles. Elle a définitivement conquis une place importante dans plusieurs domaines en recherche et développement, et en contrôle qualité. De nos jours, la plupart des mesures utilisent des diffractomètres et sont pilotées par ordinateur.

Pourtant, dans les dernières années la situation s'est troublée du fait de l'élargissement des connaissances de base et de la multiplication des problèmes et des procédés spécifiques qui en résultent. Nous allons décrire en renonçant à de nombreux détails techniques le développement historique de l'analyse des contraintes par diffraction des rayons $\mathrm{X}$ à partir des années vingt, sur la base de la découverte des rayons X en 1895 . Nous présenterons ensuite l'état actuel de cette analyse par des problèmes actuels, ainsi qu'une perspective de développement futur. On trouve dans [1] une description historique plus détaillée, tandis que [2] propose un résumé des détails techniques. Les références bibliographiques de travaux se rapportant à l'analyse des contraintes par rayons $\mathrm{X}$ ont étés rassemblées par Isenburger [3] et J. Hauk [4-10].

\section{DEVELOPPEMENT DES BASES POUR L'ANALYSE DES CONTRAINTES PAR RAYONS X ENTRE 1895 ET 1922}

Après la sensationnelle découverte des rayons X par Wilhem Conrad Röntgen le 8 novembre 1895 à Würzburg, qualifiés par Röntgen lui-même de "rayons X", ces nouvelles connaissances se sont propagées dans le monde entier à une vitesse étonnante pour l'époque. On s'est alors intéressé dans de nombreux instituts de recherche français, allemands, anglais et américains au perfectionnement des clichés par transmission. Des radiographies de mains de visiteurs furent réalisées dans le cadre de colloques scientifiques et d'expositions publiques, en montrant ainsi le côté pratique de ces rayons. La propagation soudaine de cette "nouvelle photographie" est d'autant plus compréhensible qu'elle livre aux scientifiques, et surtout aux biologistes et aux médecins, à travers des images sur des écrans fluorescents et des plaques photographiques, un contenu d'informations qu'ils avaient tenu jusqu'alors pour inaccessibles. Les figures $1 \mathrm{a}$ et $1 \mathrm{~b}$ montrent par exemple respectivement, la radiographie d'une main avec des boules de Teschin et une limande dont les boyaux sont remplis de coquillages. En raison de leur visibilité 
directe, ces images enthousiasmèrent non seulement l'esprit de recherche des scientifiques, mais donnèrent également libre court à l'imagination de toute la population. Les espérances et les inquiétudes liées à cette expérience sont bien montrées par la caricature de l'artiste Français $A$. Robida, parue dès mai 1896 dans la revue "La Nature", présentée figure 2.

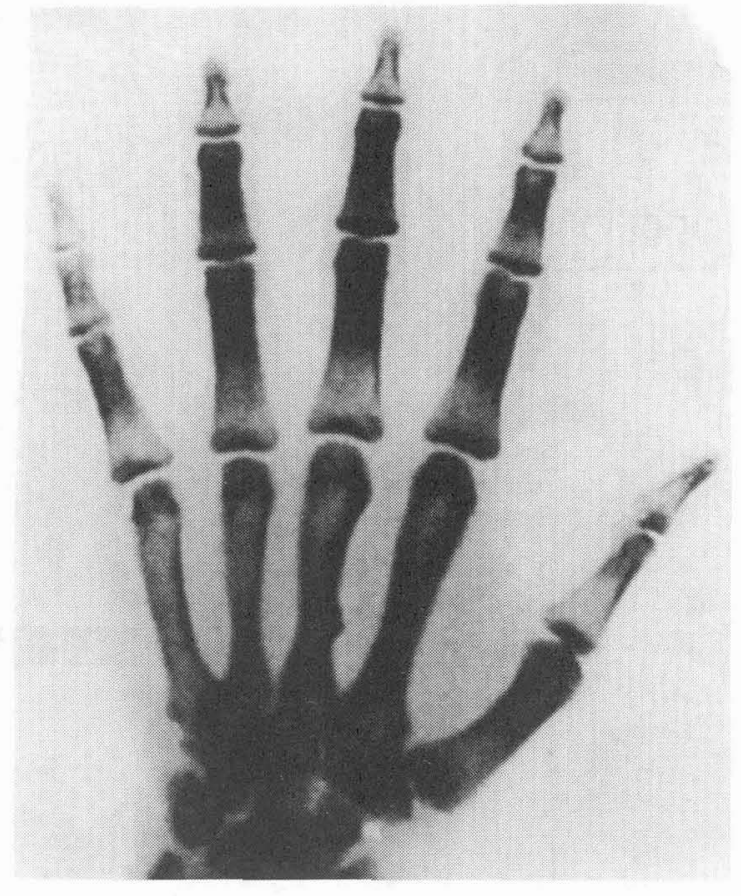

Fig. 1 : Photographies aux rayons X d'une main présentant des boules de Teschin, Musée Allemand des Rayons $\mathrm{X}$, (a) et d'une limande ayant ingéré des moules (b). 


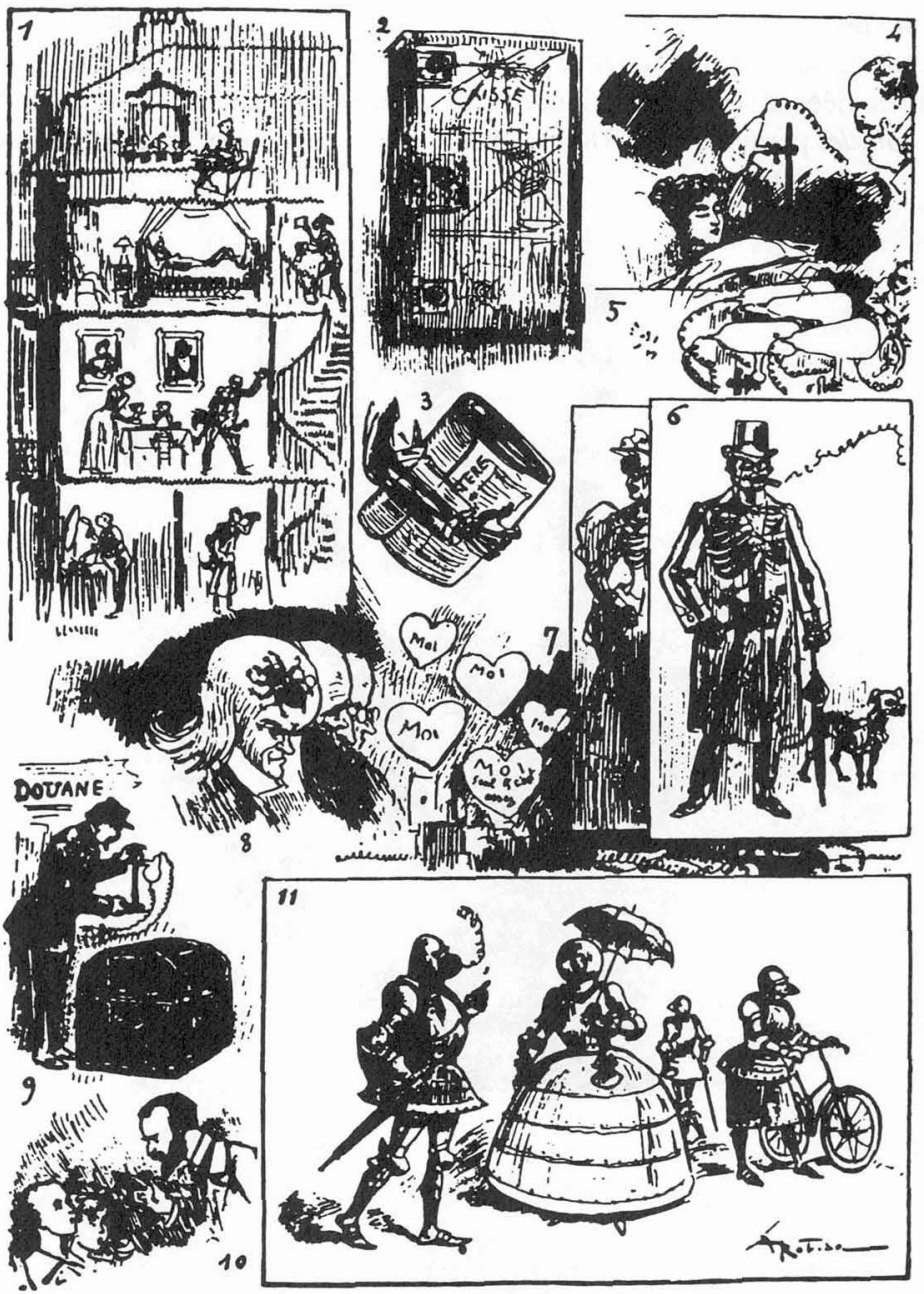

Fig. 2 : Gravures du dessinateur français A. Robida sur le thème : "Variations sur les Rayons $\mathrm{X}^{\text {" : (1) }}$ L'indiscrétion des rayons X, (2) Que contient le coffre du banquier ?, (3) Que contient la malette du ministre ?, (4) A quoi rêve l'amante ?, (5) A la recherche de la cachette des microbes, (6) Connais-toi toi-même ?, (7) Ce que contient le coeur de certains hommes, (8) Ce que contient le cerveau de certains grands savants, (9) Contrôle d'une valise à la douane, (10) Les rayons $X$ vont bien nous montrer qui a mangé le gâteau, (11) La mode de demain pour se protéger de l'indiscrétion de la nouvelle photographie.

Suite au large engouement que suscita la prise de photographie par rayons X, plus de 1000 publications sur des travaux généraux ont été réalisées durant l'année suivant la découverte des rayons $X$. Le nombre de ces travaux ne concernant que la physique et la technique resta en revanche faible, comme le montre la figure 3. Après 400 publications en 1896, leur nombre s'est fortement réduit dans les années suivantes. L'essentiel de cette recherche concernait alors l'absorption du rayonnement par différents matériaux ainsi que les propriétés ionisantes des rayons $\mathrm{X}$. Naturellement ces thèmes moins compréhensibles pour le public étaient moins spectaculaires que les radiographies des premiers jours. 


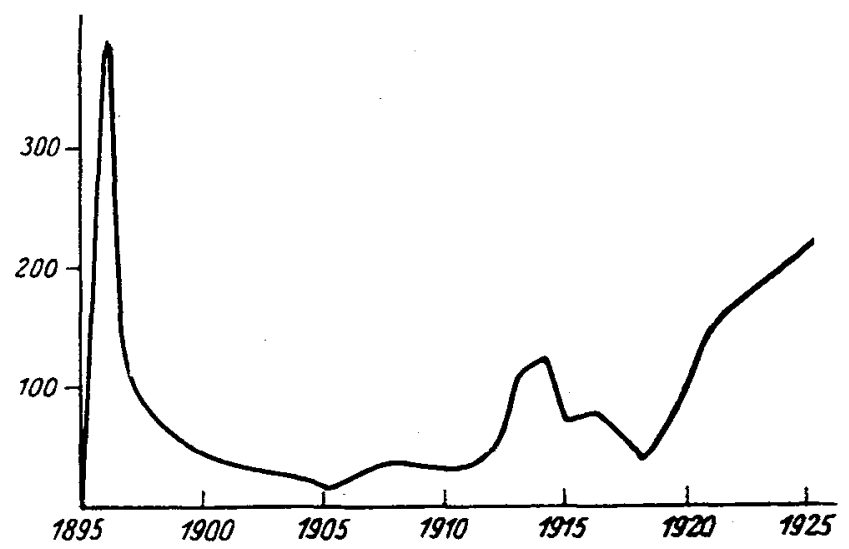

Fig. 3 : Evolution dans le temps de la littérature consacrée aux rayons X jusqu'en 1925, [1].

Les recherches physiques connurent un nouvel essor en 1912 après la découverte et la preuve de la diffraction des rayons $\mathrm{X}$ par les réseaux cristallins. Röntgen occupait alors la chaire de physique expérimentale à Munich et travaillait avec ses assistants, Brentano, Dagor, Glocker, Joffe et Wagner. Sommerfeld, avec le soutien de Röntgen, fut appelé à la chaire de physique théorique et y travailla avec ses assistants Debye, Laue, Ewald, Friedrich et Knipping. Après une discution avec Ewald, Laue supposa que si les rayons X possédaient le même caractère ondulatoire que la lumière visible, avec cependant des longueurs d'onde plus courtes, les rayons $\mathrm{X}$ feraient apparaître des phénomènes de diffraction dans les réseaux cristallins. Les professeurs Sommerfeld et Röntgen étaient sceptiques. Laue, Friedrich et Knipping furent tout de même autorisés par les professeurs à poursuivre les expériences à partir desquelles ils réalisèrent le premier cliché de Laue d'un cristal de sulfate de cuivre. La figure 4 montre par exemple une photographie de Laue d'un cristal de sulfure de zinc balayé par des rayons parallèles aux arêtes principales. La preuve de l'existence de la diffraction des rayons X par les cristaux était faite. En 1912 et 1913,W.L. Bragg et W.H. Bragg développèrent le modèle bien connu de réflexion. La position angulaire $\theta$ des maxima de diffraction est donné par la loi de Bragg:

$$
\mathrm{n} \lambda=2 \mathrm{D} \sin \theta
$$

où $\mathrm{D}$ est la distance entre deux plans de réflexion. En 1913, Laue élabora la théorie de diffraction pour les phénomènes d'interférence dans des réseaux cristallins non-primitifs. Il définit le facteur de structure qui caractérise l'intensité des interférences. Ces nouveaux outils permirent de faire avancer la recherche dans le domaine des structures cristallines. C'est pourquoi, bien qu'interrompus pendant la guerre, les travaux de recherches par rayons $\mathrm{X}$ augmentèrent de manière continue (figure 3 ). C'est ainsi que furent établies les bases de l'analyse par diffraction des rayons $\mathrm{X}$ des déformations de réseaux et des contraintes. 


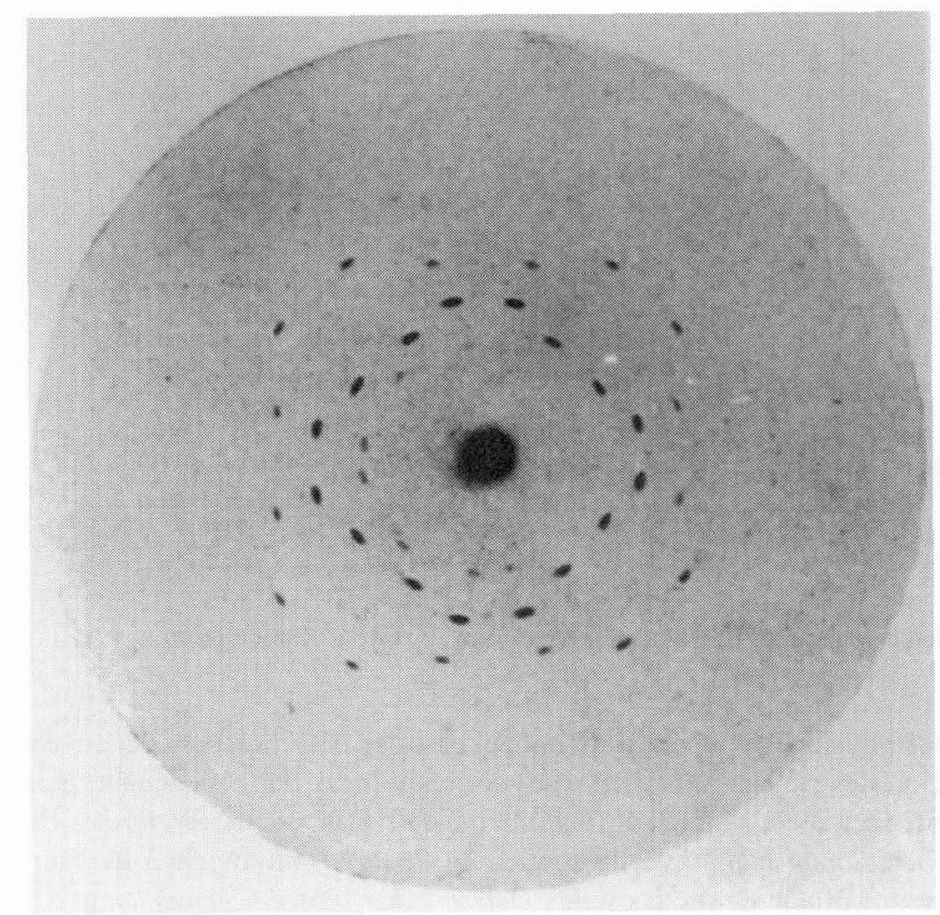

Fig. 4 : Photographie de Laue d'un cristal de sulfure de zinc balayé par des rayons parallèles aux arêtes principales, [1].

\section{PREMIERE ANALYSE PAR RAYONS X DES DEFORMATIONS DANS UN RESEAU ET DES CONTRAINTES, ET DESCRIPTION DU PRINCIPE FONDAMENTAL DE L'ANALYSE DES CONTRAINTES PAR RAYONS $X$ ENTRE 1922 ET 1945}

Joffê et Kirpitcheva réalisèrent en 1922 à l'institut de Saint Pétersbourg qui est encore aujourd'hui appelé Institut Joffè, les premières analyses de déformations par rayons X. Il utilisa le montage schématisé sur la figure 5.. A l'aide d'un électroaimant, il soumit un monocristal de chlorure de sodium à une contrainte bien définie. Il constata que les points d'interférence se déplaçaient par rapport à l'état sans contrainte, figure 5 . Il en déduisit l'existence de déformations élastiques du réseau cristallin [11]. 


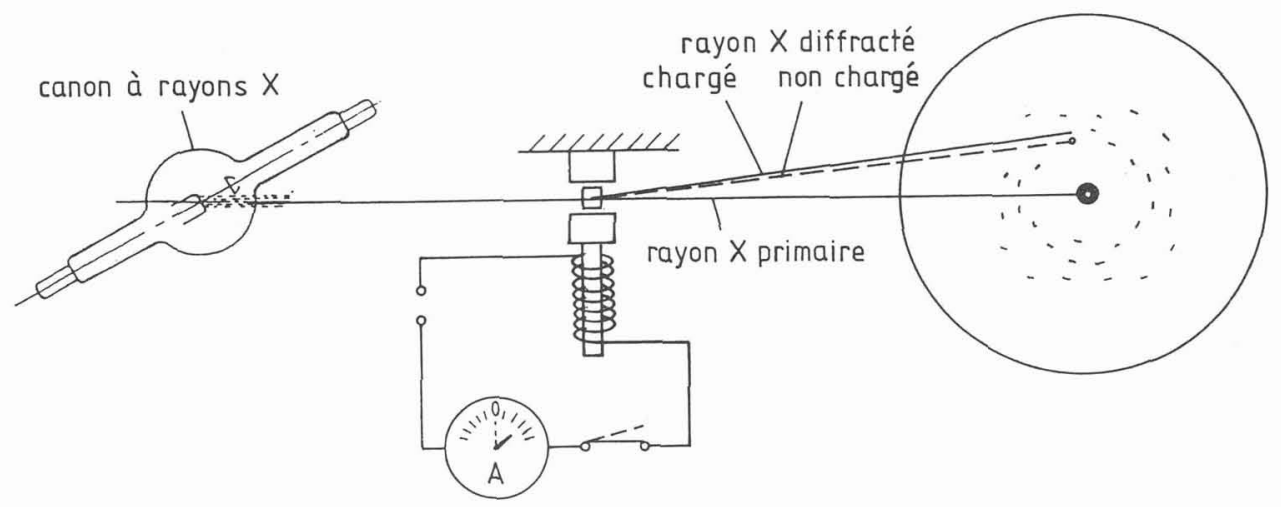

Fig. 5 : Montage expérimental pour la réalisation de photographies de Laue d'un cristal salin chargé à l'aide d'un électroaimant.

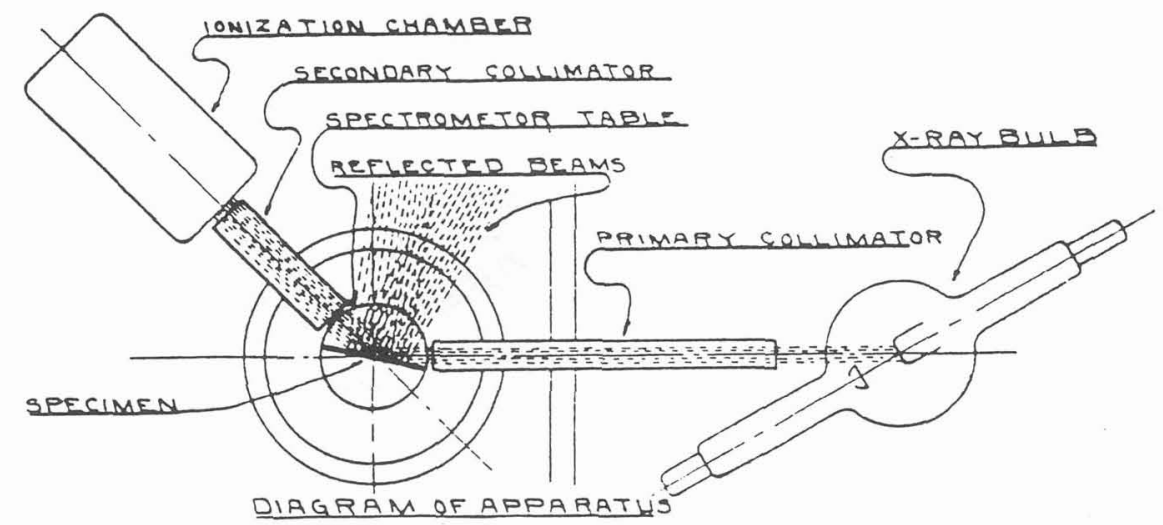

Fig. 6 : Schéma d'un montage expérimental pour l'enregistrement d'interférences des rayons $X$ à l'aide d'un collimateur de Soller, utilisant un spectromètre de Bragg et une chambre d'ionisation.

En 1924 à Cincinnati, Soller déterminait les constantes de réseau, la dilatation thermique et les déformations de réseau dans un cristal soumis à des contraintes extérieures [12]. Il utilisa un spectromètre de Bragg permettant de positionner l'échantillon et une chambre à ionisation jouant le rôle de détecteur en les tournant autour du même axe. Cet ensemble permit l'obtention de données quantitatives avec une bonne précision en utilisant un collimateur composé de fentes étroites (figure 6). Il est encore appelé aujourd'hui "collimateur de Soller". Les rayons émis par le canon à rayons $\mathrm{X}$ arrivent parallèles sur la surface de l'échantillon et l'intensité des rayons diffractés est enregistrée par la chambre d'ionisation, un collimateur de Soller placé devant celle-ci. Il se rendit compte que les déformations de réseau dues à une contrainte externe étaient de l'ordre de grandeur des déformations macroscopiques.

Le goniomètre à compteur construit et utilisé par Soller ne fut pas mis en pratique, à cause de son encombrement. D'autre part les lamelles relativement grossières du collimateur causaient des difficultés d'identification des lignes d'interférences enregistrées. Suite à cela, on construisit des chambres photographiques pour enregistrer les lignes d'interférences lors de l'analyse de contraintes par rayons X. Dehlinger [13] développa en 1927 une chambre où le rayon incident passe par des fentes avant d'atteindre l'échantillon monté sur la partie arrière 
(figure. 7). Il est ainsi possible d'obtenir avec une bonne précision les déformations du réseau parce que les angles de diffraction sont grands. La surface intéressante de l'échantillon peut être placée de manière bien reproductible sur la partie arrière de la chambre. Les rayons diffractés sont enregistrés sur un film de forme arrondi placé le long de la paroi de la chambre. En 1929, le Russe Aksenow, se basant sur le modèle de Bragg, décrivit le principe fondamental de l'analyse de contraintes par diffraction des rayons $\mathrm{X}$ sur des matériaux polycristallins [14]. Il enregistre des anneaux d'interférences de matériaux polycristallins et en comparant la position $\theta$ de ces lignes avec celle d'un matériau soumis à aucune contrainte $\theta_{\mathrm{o}}$, il détermina les déformations

$$
\varepsilon=-\left(\theta-\theta_{0}\right) \cot \theta_{0}
$$

du réseau dans la direction du vecteur de diffraction. Pour un polycristal, on se place dans un cristallite d'orientation définie et on y détermine les déformations du réseau perpendiculaire aux plans cristallins de type $\{\mathrm{hkl}\}$. Le principe de l'analyse de contraintes par rayons $\mathrm{X}$ repose sur le fait qu'on peut identifier les déformations du réseau obtenues dans des directions données aux déformations macroscopiques présentes. On peut calculer les valeurs de contraintes en faisant des hypothèses sur les états de contraintes et la théorie de l'élasticité.

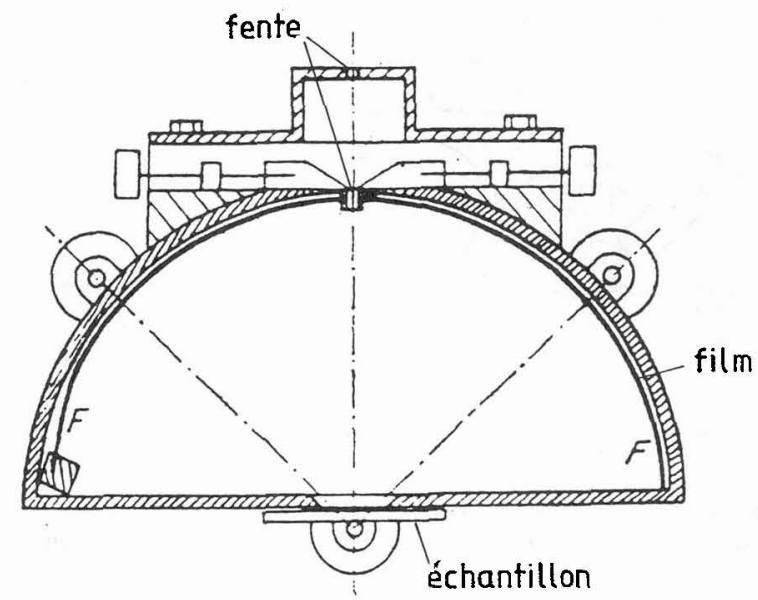

Fig. 7 : Chambre photographique pour l'enregistrement d'interférences de rayons réfléchis en retour d'après Dehlinger.

En 1930, Sachs et Weerts appliquèrent le procédé des rayons diffractés en retour sur une plaque d'un alliage d'aluminium soumis à une flexion [15]. Ils déterminèrent ainsi la variation des distances entre plans cristallins quasi-parallèles à la surface de l'échantillon (figure 8a). On peut voir sur la photographie les lignes d'interférences des plans $\{511 / 333\}$ obtenues pour différentes flexions $\mathrm{f}$ de l'échantillon avec un rayonnement $\mathrm{Cu} K \alpha$. Les variations des constantes du réseau dépendent de la flexion de la tôle et par conséquent de la contrainte théorique à la surface de l'échantillon (fígure 8b). 


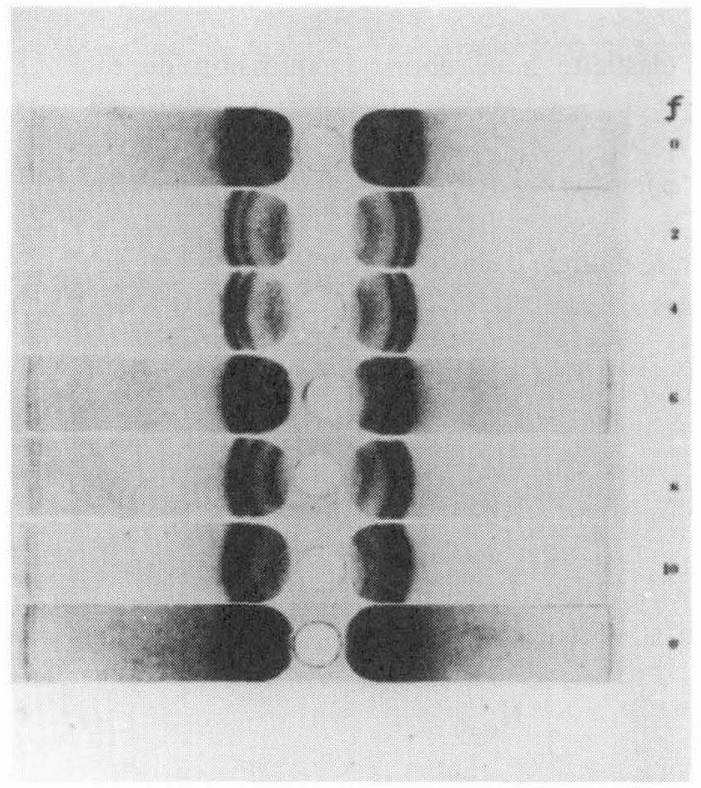

a)

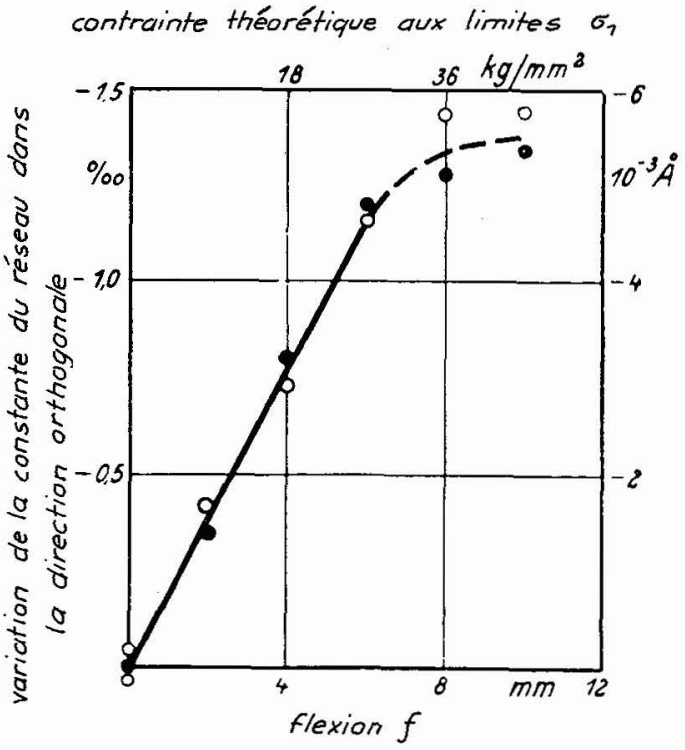

b)

Fig. 8 : Lignes d'interférences des plans $\{511 / 333\}$ obtenues avec des rayonnements $\mathrm{Cu} \mathrm{K} \alpha$ pour différentes flexions $f(a)$ et la variation des constantes de réseau dans la direction normale à la surface en fonction de la flexion $\mathrm{f}(\mathrm{b})$.

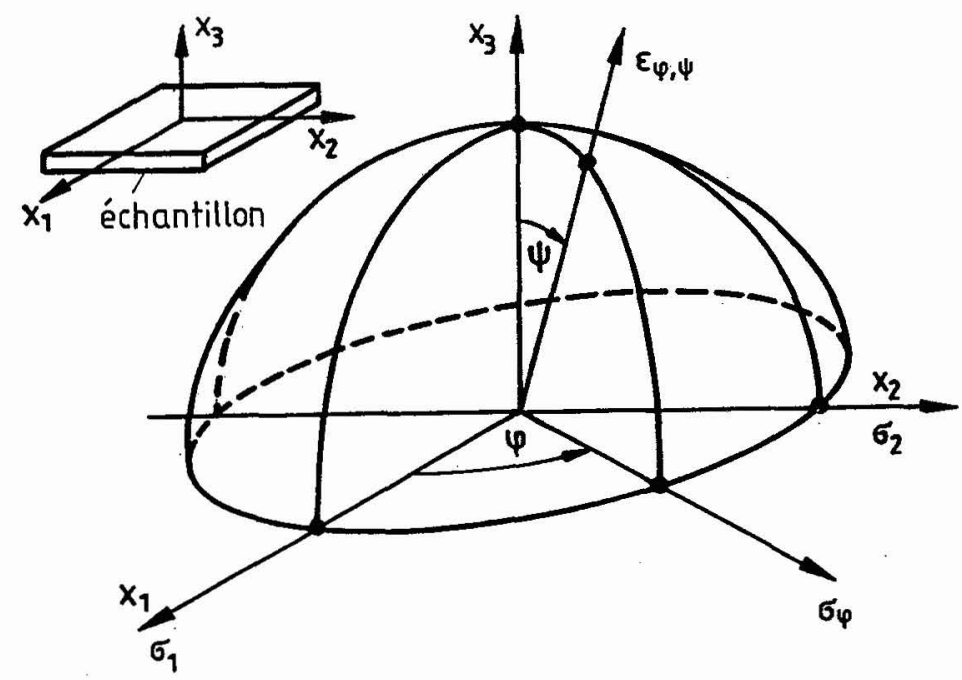

Fig. 9 : Système de coordonnées et description des angles.

Il est nécessaire de connaître quelques bases de la théorie de l'élasticité pour comprendre ce phénomène. Considérons un état de contraintes plan dans un polycristal de contraintes principales $\sigma_{1}$ et $\sigma_{2}$ proche de la surface et parallèle à celle-ci (voir le système de 
coordonnées sur la figure 9). La théorie de l'élasticité nous donne l'expression de la déformation

$$
\varepsilon_{\varphi, \psi}=\frac{1+\nu}{E}\left(\sigma_{1} \cos ^{2} \varphi+\sigma_{2} \sin ^{2} \varphi\right) \sin ^{2} \psi-\frac{\nu}{E}\left(\sigma_{1}+\sigma_{2}\right)
$$

dans la direction définie par les angles $\varphi$ et $\psi$. E est le module d'élasticité et $v$ le coefficient de Poisson. La contrainte normale suivant l'azimut $\varphi$ est

$$
\sigma_{\varphi}=\sigma_{1} \cos ^{2} \varphi+\sigma_{2} \sin ^{2} \varphi
$$

A l'aide des constantes élastiques de Voigt

on obtient la relation

$$
\frac{1}{2} S_{2}=\frac{1+\nu}{E} \text { et } S_{1}=\frac{-v}{E}
$$

$$
\varepsilon_{\varphi, \psi}=\frac{1}{2} S_{2} \sigma_{\varphi} \sin ^{2} \psi-S_{1}\left(\sigma_{1}+\sigma_{2}\right)
$$

a)

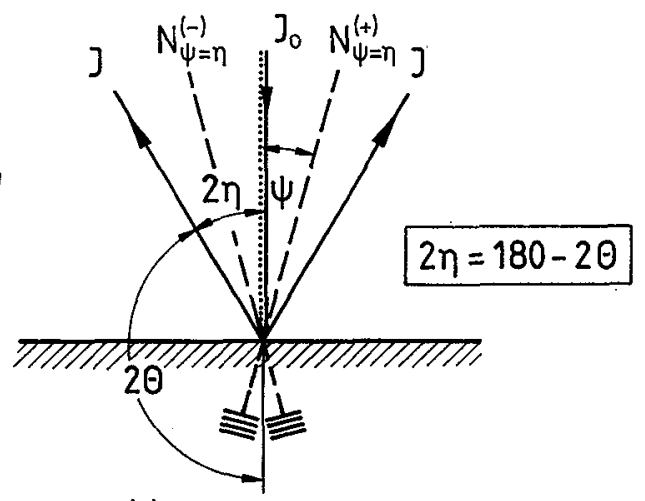

c)

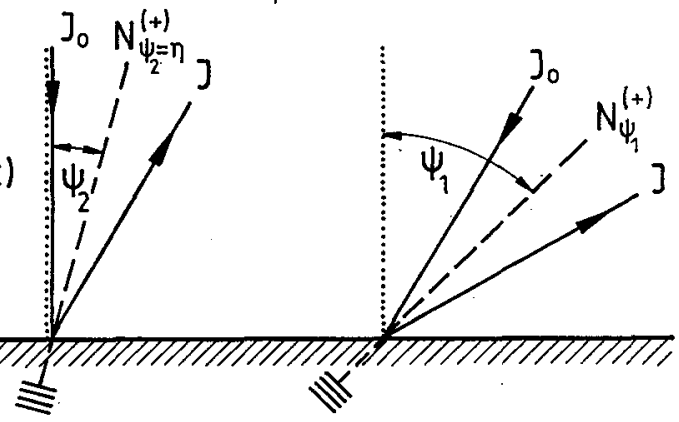

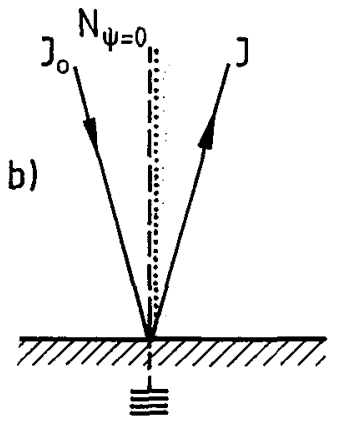

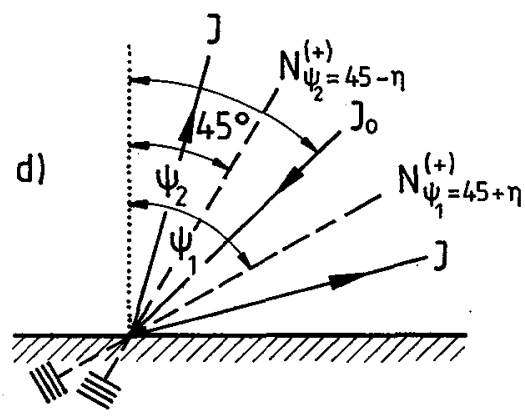

Fig. 10 : Trajectoire des rayons (Io : rayon incident, I : rayon réfléchi) pour la mesure des lignes d'interférences des plans du réseau de normale $\mathrm{N}$ sous plusieurs angles $\psi$ par rapport à la normale à la surface. (a) méthode du rayon incident perpendiculaire, (b) méthode du rayon incident incliné, (c) méthode de mesure avec un rayon incident perpendiculaire et un incident incliné, (d) méthode avec un rayon incident incliné d'un angle de $45^{\circ}$.

La schématisation de la trajectoire des rayons réalisée par Sachs et Weerts est représentée sur la figure $10 \mathrm{a}$. On considère un rayonnement incident $I_{0}$ perpendiculaire à l'échantillon, diffracté par les plans de normale $\mathrm{N}$ et de distance interréticulaire $\mathrm{D}$ qui sont sensiblement 
parallèles à la surface de l'échantillon. On enregistre les déformations du réseau dans la direction du demi-angle entre les rayonnements incident $\mathrm{I}_{0}$ et diffracté $\mathrm{I}$, c'est à dire sous un angle $\eta=90^{\circ}-\theta$ par rapport à la normale de l'échantillon. Comme l'angle $\eta$ est relativement faible, on le remplace dans l'équation (6) par $\psi=\eta \approx 0^{\circ}$ et on obtient

$$
\mathcal{E}_{\varphi, \psi \approx 0^{\circ}}=\frac{D_{\varphi, \psi \approx 0^{\circ}}-D_{o}}{D_{o}} \approx S_{1}\left(\sigma_{1}+\sigma_{2}\right)
$$

$\mathrm{D}_{\mathrm{o}}$ est la distance entre plans soumis à aucune contrainte externe. On ne peut évidemment obtenir que la somme des contraintes principales. Dans le cas précédant de la flexion uniaxiale, on a $\sigma_{2}=0$. La variation de déformation est donc égale à la variation de la contrainte de flexion $\sigma_{1}$.

En 1931, Wever et Möller améliorèrent la méthode appliquée par Sachs et Weerts pour déterminer les déformations du réseau sur des plans parallèles à la surface, c'est à dire dans la direction perpendiculaire à la surface de l'échantillon (figure 10b) [16]. Pour un angle d'incidence $\eta$ par rapport à la normale à la surface de l'échantillon et pour $\psi=0^{\circ}$, la déformation du réseau est

$$
\varepsilon_{\varphi, \psi=0^{\circ}}=\frac{\mathrm{D}_{\varphi, \psi=0^{\circ}}-\mathrm{D}_{\mathrm{o}}}{\mathrm{D}_{\mathrm{o}}}=\mathrm{S}_{1}\left(\sigma_{1}+\sigma_{2}\right)
$$

Parallèlement, Wever et Möller développèrent en 1932 une chambre photographique (figure 11a) [17] dont le rayonnement $X$ incident est localisé par une fente étroite et irradie un large domaine de l'échantillon. Le rayonnement réfléchi est compris dans un cône. L'intersection du cône et du film, et la fente sont localisés sur une sphère autour du point irradié sur la surface de l'échantillon. Ainsi, les rayons réfléchis sont localisés sur le film par des raies de diffraction très fortes et contrastées. Le film est stocké sous forme de rouleaux qui permettent de le faire tourner autour de l'axe du rayon incident. Pour cette raisons les matériaux cristallins à gros grains forment, en tournant le film, des anneaux d'interférences. Pour déterminer la position des raies diffractées, la distance entre le film et la surface de l'échantillon doit être parfaitement connue. En 1933, Wever et Möller proposèrent une méthode pour déterminer cette distance [18]. Ils enregistrèrent sur le même film des raies de l'échantillon et d'un matériau de référence sans contrainte ayant une constante de réseau connue (figure 11b).
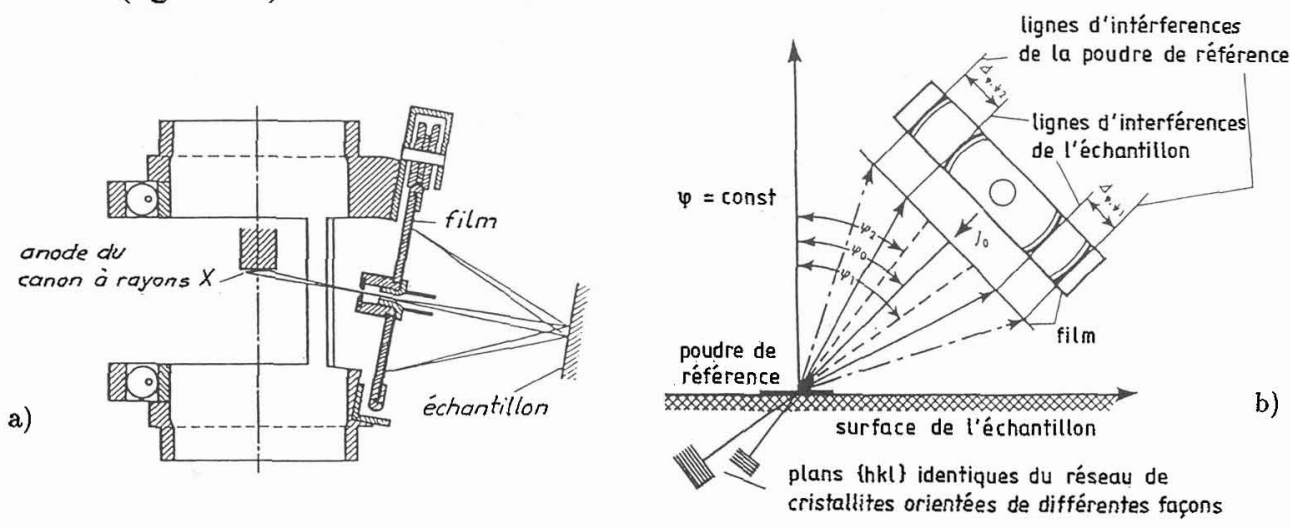

Fig. 11 : Chambre photographique dont les rayons sont localisés avec film tournant (a) et comportements géométriques inclinés pour un cliché en retour d'un échantillon recouvert d'une poudre de référence (b). 
En 1935, Glocker et Osswald [19] firent des progrès essentiels en développant un procédé pour obtenir une seule des composantes normales $\sigma_{\varphi}$. Ils firent deux photographies, l'une avec un rayonnement incident $I_{o}$ normal et l'autre avec un rayonnement incident incliné par rapport à la normale à la surface (figure $10 \mathrm{c}$ ). La différence entre les deux déformations obtenues pour $\psi \approx 0^{\circ}$ et $\psi$ est

$$
\varepsilon_{\varphi, \psi}-\varepsilon_{\varphi, \psi} \approx 0^{\circ}=\frac{D_{\varphi, \psi}-D_{\varphi, \psi} \approx 0^{\circ}}{D_{o}} \approx \frac{1}{2} S_{2} \sigma_{\varphi} \sin ^{2} \psi
$$

d'où la contrainte normale

$$
\sigma_{\varphi}=\frac{\varepsilon_{\varphi, \psi}-\varepsilon_{\varphi, \psi \approx 0^{\circ}}}{\frac{1}{2} S_{2} \sin ^{2} \psi}
$$

suivant l'azimut $\varphi$ et l'inclinaison $\psi$. Un autre avantage est qu'on peut bien exprimer la différence entre les deux déformations par l'approximation

$$
\frac{D_{\varphi, \psi}-D_{\varphi, \psi} \approx 0^{\circ}}{D_{o}} \approx \frac{D_{\varphi, \psi}-D_{\varphi, \psi} \approx 0^{\circ}}{D_{\varphi, \psi} \approx 0^{\circ}}
$$

Ainsi l'expression de la contrainte suivant l'équation (10) est pratiquement indépendante de la distance $\mathrm{D}_{\mathrm{o}}$, qui est difficile à déterminer expérimentalement.

En 1938, Glocker et col. [20] réussirent à déterminer la composante de la contrainte $\sigma_{\varphi}$ en ne réalisant qu'une seule photographie et sans avoir besoin de la distance $D_{0}$. Ils n'utilisèrent pour cela qu'un rayonnement incident incliné par rapport à la normale suivant l'azimut $\varphi$. Ils ont alors enregistré deux rayonnements diffractés symétriquement sur le cône d'interférence (figure 10d). Ils obtinrent ainsi les déformations du réseau dans les deux directions $\psi_{1}$ et $\psi_{2}$. D'où la connaissance de $\sigma_{\varphi}$, par l'intermédiaire de l'équation (9)

$$
\varepsilon_{\varphi, \psi_{1}}-\varepsilon_{\varphi, \psi_{2}}=\frac{1}{2} S_{2} \sigma_{\varphi}\left(\sin ^{2} \psi_{1}-\sin ^{2} \psi_{2}\right)
$$

Le travail de Romberg [21] est également remarquable. Il discuta en 1937 du problème suivant, comment les états de contraintes triaxiaux dans des domaines de matériaux irradiés influencent les déformations du réseau mesurés par rayons $\mathrm{X}$. De ce fait, il constata que six clichés étaient nécessaires pour déterminer les six composantes inconnues d'un état de contrainte orienté de façon quelconque

$$
\left(\sigma_{i j}\right)=\left(\begin{array}{lll}
\sigma_{11} & \sigma_{12} & \sigma_{13} \\
\sigma_{12} & \sigma_{22} & \sigma_{23} \\
\sigma_{13} & \sigma_{23} & \sigma_{33}
\end{array}\right)
$$


Il fournit pour cela la relation

$$
\begin{aligned}
\varepsilon_{\varphi, \psi} & =\frac{1}{2} S_{2}\left[\sigma_{11} \cos ^{2} \varphi+\sigma_{12} \sin (2 \varphi)+\sigma_{22} \sin ^{2} \varphi-\sigma_{33}\right] \sin ^{2} \psi \\
& +\frac{1}{2} S_{2}\left[\sigma_{13} \cos \varphi+\sigma_{23} \sin \varphi\right] \sin (2 \psi) \\
& +\left(\frac{1}{2} S_{2}+S_{1}\right) \sigma_{33}+S_{1}\left(\sigma_{1}+\sigma_{2}\right)
\end{aligned}
$$

Un problème essentiel dans l'analyse des contraintes par rayons $X$ survint rapidement. Les contraintes mesurées $\sigma^{\text {rayons } X}$ se révélèrent différentes des contraintes mécaniques induitent par les charges extérieures. Avec un rayonnement Co K $\alpha$ irradiant les plans $\{311\}$ ou un rayonnement $\mathrm{Cr} \mathrm{K} \alpha$ irradiant les plans $\{211\}$ on obtient respectivement des valeurs de contraintes $\sigma^{\text {rayons } X}$ plus grandes ou plus petites que les contraintes mécaniques appliquées. En 1935, Möller et Barbers purent expliquer les raisons de cette différence par l'anisotropie élastique des cristallites des matériaux polycristallins [22]. Ainsi, les déformations de réseau de cristaux spéciaux pour lesquels les normales aux plans $\{\mathrm{hkl}\}$ se trouvent dans la direction $\mathrm{du}$ vecteur de diffraction sont toutes différentes des véritables déformations macroscopiques. Ce constat montra que l'hypothèse d'homogénéité des déformations dans tous les cristallites d'un matériau polycristallin de Voigt [23] était erronée. Les effets d'anisotropie furent d'abord introduits empiriquement par la grandeur

$$
\xi=\frac{\sigma^{\text {rayons } \mathrm{X}}}{\sigma^{\text {mé canique }}}
$$

Pour les plans $\{310\}$ de la ferrite soumis à un rayonnement Co $\mathrm{K} \alpha$, on détermina un "module d'élasticité radiocristallographique" inférieur d'environ $8 \%$ à la valeur mécanique.

Le calcul des constantes élastiques fondé sur l'hypothèse de Reuss [24] permit un progrès important dans l'analyse des contraintes par rayons X. Celui-ci supposa l'homogénéité des contraintes dans tous les cristallites. Il en résulte, comme le montrèrent Glocker et Schiebold en 1938 [25, 26], une dépendance des constantes élastiques radiocristallographiques et des plans $\{\mathrm{hkl}\}$. Ces constantes permirent d'obtenir la tendance de l'évolution des contraintes en fonction des plans $\{\mathrm{hkl}\}$, mais les valeurs des contraintes ne correspondaient toujours pas aux contraintes appliquées.

Ce n'est qu'en 1941 que Möller et Neerfeld proposèrent une méthode empirique [27] qui consiste à utiliser une valeur moyenne des calculs de Voigt et Reuss pour les constantes élastiques radiocristallographiques.

En raison des progrès méthodiques précédants dans l'analyse des contraintes par diffraction des rayons $\mathrm{X}$ et après que leur usage se fut révélé bonne pour la détermination de contraintes externes, on se servit de l'analyse des contraintes par rayons $\mathrm{X}$ pour la détermination des contraintes résiduelles liées à des déformations inhomogènes élastoplastiques. Suite aux essais de Heyn (1921) [28] et Masing (1925) [29], on a différencié les contraintes résiduelles macroscopiques (type I), qui sont homogènes dans des grands domaines du matériau, des contraintes résiduelles microscopiques (type II ou III) qui sont distribuées de façon homogène ou inhomogène dans des plus petits domaines. On considérait alors que, seules les contraintes résiduelles macroscopiques équivalentes aux contraintes externes conduisaient aux déplacements des lignes d'interférences. Les contraintes résiduelles macroscopiques, par contre, conduisaient à leur élargissement. 
A partir de ces travaux, Norton étudia en 1932 les contraintes résiduelles dues aux soudures [30] et en 1937 leur relaxation thermique [31], Wever et Möller étudièrent en 1933 les contraintes résiduelles de flexion [17] et, Wassermann et Romberg en 1935 et 1937 les contraintes résiduelles dues à la trempe $[32,21]$. En 1938, Nishihara et Kojima étudièrent les contraintes résiduelles dues en présence de fonte [33] ainsi que Glocker et col.. [34] les variations de contraintes résiduelles à la suite de sollicitations périodiques en flexion. Krächter détermina en 1939 au moyen des rayons X des coefficients d'entaille [35]. En 1940, Altschuler et Speranski firent part des contraintes résiduelles d'usinage [36] ainsi que Mahla des contraintes résiduelles dans des couches galvanisées [37]. Finalement, Milburn vérifia en 1949 la distribution des contraintes résiduelles liées au grenaillage [38]. Beaucoup de questions qui font encore aujourd'hui l'objet de recherche et d'intérêt ont été posées dès les années 30 .

\section{DEVELOPPEMENT ET APPLICATIONS DES ANALYSES PAR RAYONS X AUX DEFORMATIONS DE RESEAU ET AUX CONTRAINTES ENTRE 1945 ET 1958}

La période entre 1945 et 1958 fut caractérisée par deux tendances de développement contradictoires. D'une part, l'analyse de contraintes par rayons $\mathrm{X}$ connut des développements essentiels aussi bien dans les méthodes que dans l'instrumentation. Mais d'autre part, des objections grandirent quant à leurs applications sans limite et des questions fondamentales surgirent quant à l'interprétation des résultats.

Tout d'abord, les expériences se poursuivirent suivant les procédés classiques décrits cidessus. Cela semble d'autant plus justifié que ces méthodes ont prouvé leur fiabilité et leur utilité dans la détermination de contraintes externes connues. Ainsi Bollenrath et Hauk découvrirent en 1946 les influences structurales sur l'analyse des contraintes par rayons X [39], de même que Norton et col. [40] l'influence des contraintes résiduelles de compression sur la résistance à la fatigue des échantillons entaillés. Osswald discuta en 1948 des effets de gradients de contraintes sur l'analyse des contraintes par rayons X [41]. Frohnmeyer et Hofmann firent des expériences en 1952 avec le procédé de rayons X en retour pour l'analyse des déformations de cristallites individuelles ferritique dans des aciers sollicités en traction [42].

Partant des travaux de Durer de 1946 [43], Glocker fournit en 1951 une contribution méthodique importante. Il montra, à l'aide de prise de photographies en réflexion en retour sur un matériau soumis à des contraintes, comment on pouvait tirer des renseignements sur une composante de contrainte $\sigma_{\varphi}$, et sur la distance interréticulaire $D_{0}$ des plans $\{h \mathbf{h l}\}$ soumis à aucune contrainte [44]. Binder et Macherauch déterminèrent en $1955 \mathrm{D}_{0}$ en considérant l'ensemble des directions sans déformation pour des états de contrainte biaxiaux [45].

Dans les années 50 surgit un brutal bouleversement dans le domaine de l'appareillage. Möller et Neerfeld montrèrent en 1948 les avantages essentiels de l'enregistrement avec des compteurs proportionnels [46]. La figure 12 montre à gauche les interférences des plans $\{310\}$ de la ferrite soumise à un rayonnement Co $\mathrm{K} \alpha$ et à droite les interférences des plans $\{211\}$ de ferrite soumise à un rayonnement $\mathrm{Cr} \mathrm{K} \alpha$. Il est évident que la détermination de la positon des raies en utilisant le compteur (figure 12a) est plus simple et plus précise qu'en utilisant des photographies (figure 12b) ou qu'à l'aide d'un photomètre (figure 12c). Au lieu d'avoir recours à l'enregistrement de films à l'arrière d'une chambre, c'est la méthode d'enregistrement par compteur avec un diffractomètre qui s'imposa. Les problèmes de contraintes résiduelles purent alors être traités pour des aciers martensitiques. En raison de la 
grande largeur des raise, leur traitement était difficile, voire impossible en utilisant l'enregistrement par film.

a)
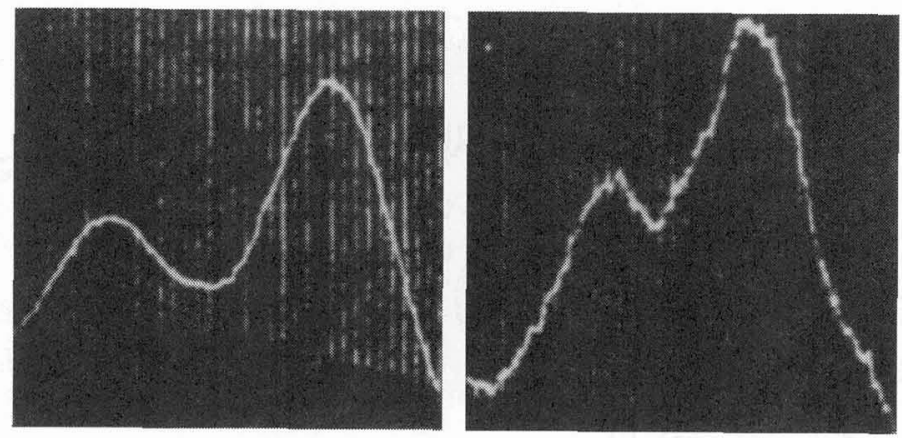

b)
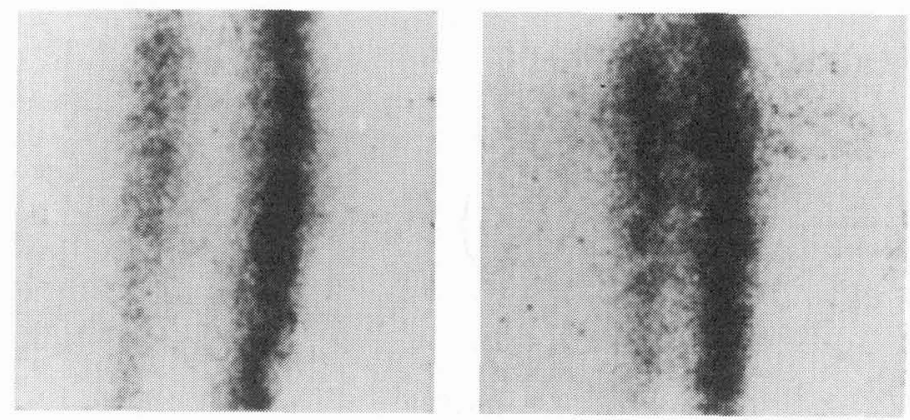

c)
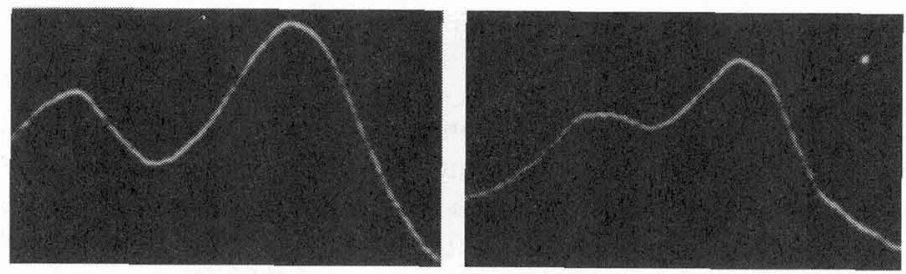

Fig. 12 : Interférences de ferrite d'acier enregistrées pendant exposition à un rayonnement $\mathrm{Co} \mathrm{K} \alpha$ (à gauche) et à un rayonnement $\mathrm{Cr} \mathrm{K} \alpha$ (à droite) avec compteur proportionnel (a), film (b) et photomètre (c).

Des travaux avec des compteurs proportionnels ont été présentés par Maloof et Erard en 1952 [47], Christenson et Rowland 1952/53 [48] et aussi par Möller et Hauk en 1955 [49].

$\mathrm{Au}$ début furent utilisés des diffractomètres Bragg-Brentano à géométrie $\Omega$. Pour ces diffractomètres, la surface de l'échantillon est inclinable seulement autour de l'axe $\theta / 2 \theta$ par rapport à la direction du rayonnement primaire fixe. On peut positionner l'échantillon, en le tournant autour de cet axe, sous différents angles $\psi$ (figure 13a). Pour l'enregistrement des raies on tourne l'échantillon à une vitesse angulaire donnée autour de l'axe du diffractomètre, le compteur tournant à une vitesse double. L'intensité du rayonnement est alors enregistrée en fonction de $2 \theta$ par une table traçante. En 1957, Möller et Brasse utilisèrent pour la première 
fois un compteur à scintillation pour enregistrer les raies diffractées [50]. Ce détecteur permettait essentiellement d'atteindre un taux de comptage plus élevé que les compteurs proportionnels.

axe $2 \theta$

axe $2 \theta$

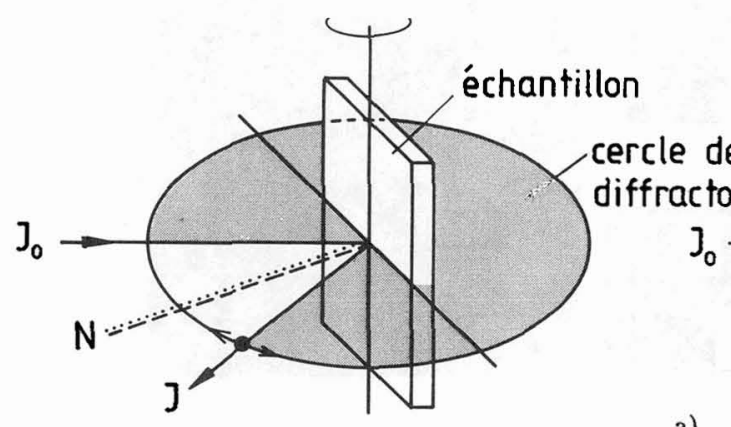

a)
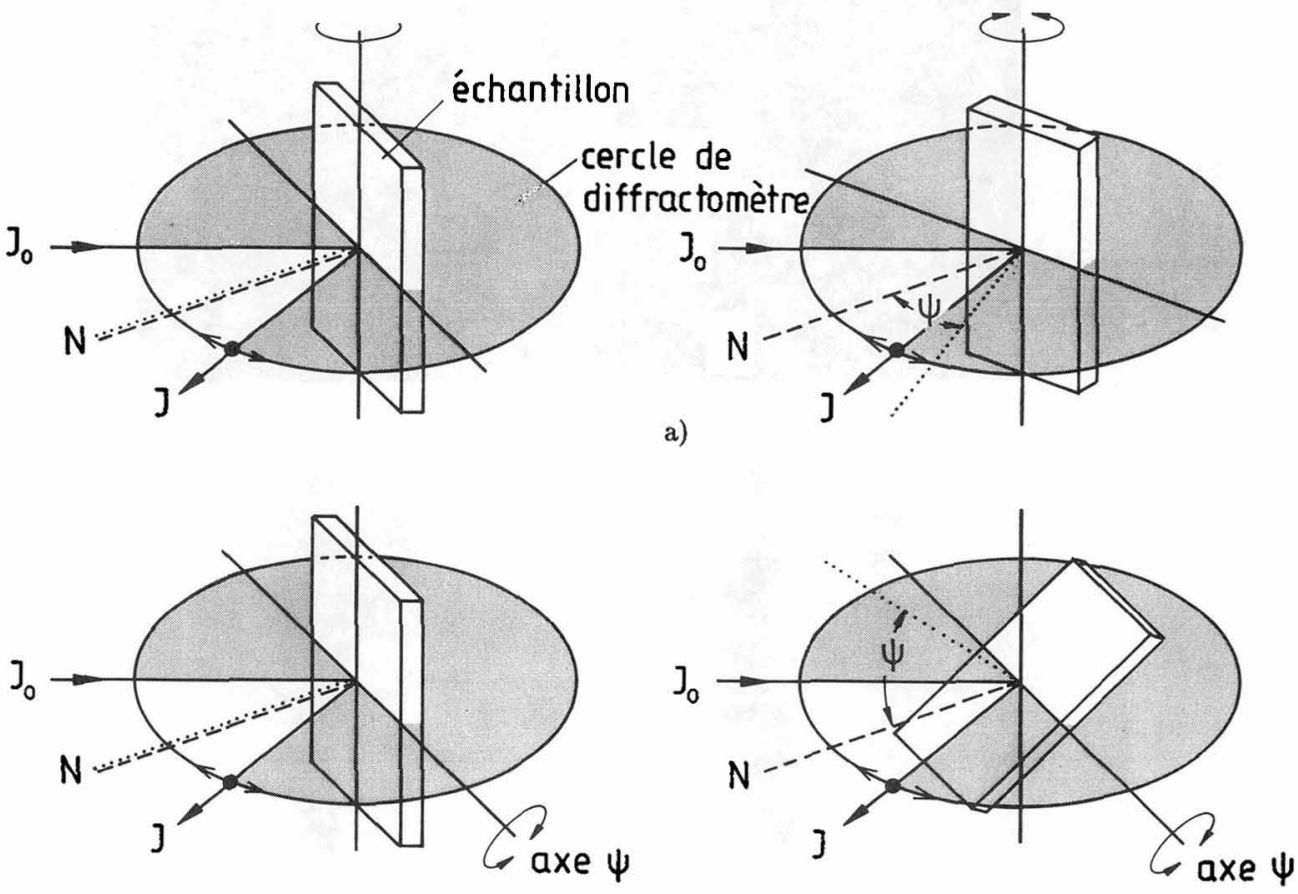

b)

Fig. 13 : Schéma du principe de montage d'un diffractomètre $\Omega$ (a) et d'un diffractomètre $\Psi$ (b).

Malgré ces progrès techniques dans l'analyse des contraintes par rayons $\mathrm{X}$, il restait de nombreux points obscurs qui pouvaient remettre en question la validité des résultats. Certaines incertitudes persistaient quant à l'influence de l'anisotropie élastique sur les valeurs des contraintes obtenues par rayons X. Le développement de l'analyse des contraintes par rayons $\mathrm{X}$ fut moins important que ce qui était prévisible. D'une part les expériences étaient difficiles à réaliser, d'autre part les procédures de mesure et d'analyse des résultats restaient troubles. Greenough [51] fit remarquer en 1949 qu'il existait une limitation encore plus importante. Jusqu'à cette époque, on supposait que l'on ne déterminait que les contraintes résiduelles de type I (contraintes résiduelles macroscopiques). Greenough a en fait montré que les contraintes résiduelles microscopiques (contraintes résiduelles de type II) peuvent aussi, sous certaines conditions dans des matériaux, provoquer des déplacements des raies diffractées. Surtout pour des matériaux plastiquement déformés, la détermination des contraintes résiduelles suivant différentes méthodes fournissaient des valeurs contradictoires, ayant parfois des signes opposés. Dans certains cas les contraintes résiduelles de déformation obtenues par l'analyse des contraintes par rayons $\mathrm{X}$ étaient à l'évidence différentes des contraintes résiduelles de type I. Ces problèmes aboutirent à une incertitude de principe quant 
à l'exploitation des résultats expérimentaux sur des matériaux homogènes ou hétérogènes ayant des contraintes résiduelles.

\section{DEVELOPPEMENT ET APPLICATIONS DES ANALYSES PAR RAYONS X AUX DEFORMATIONS DE RESEAU ET AUX CONTRAINTES ENTRE 1958 et 1980}

Les problèmes cités ci-dessus quant à l'exploitation des résultats par l'analyse de contraintes par rayons X conduisirent en 1958 au développement du procédé $\sin ^{2} \psi$ par Macherauch [52, 53]. Pour des états de contraintes résiduelles macroscopiques biaxiaux parallèles à la surface on s'attend à une proportionnalité entre les déformations de réseau $\varepsilon_{\varphi, \psi}$ et $\sin ^{2} \psi$, pour un azimut $\varphi$ donné (figure 14). La pente de cette courbe permet de déterminer la composante de la contrainte normale $\sigma_{\varphi}$, parallèle à la surface. L'avantage essentiel de ce procédé pour éliminer les incertitudes décrites ci-dessus est qu'on peut tracer les déformations du réseau en fonction de $\sin ^{2} \psi$ pour un grand domaine de $\psi$. La pente de la droite obtenue par régression linéaire fournit un résultat de contrainte statistiquement sûr et bien reproductible. A partir des écarts des points expérimentaux par rapport au comportement linéaire, on peut conclure à l'existence de textures dues aux déformations ainsi que de contraintes de type II. Les conditions expérimentales peuvent être choisies de telles façons qu'elles n'aient que peu d'influence sur les valeurs obtenues des contraintes résiduelles d'ordre I. Alors que jusqu'à présent de nombreuses mesures n'étaient exploitables que de manière insatisfaisantes, ce procédé permet d'obtenir de bons résultats.

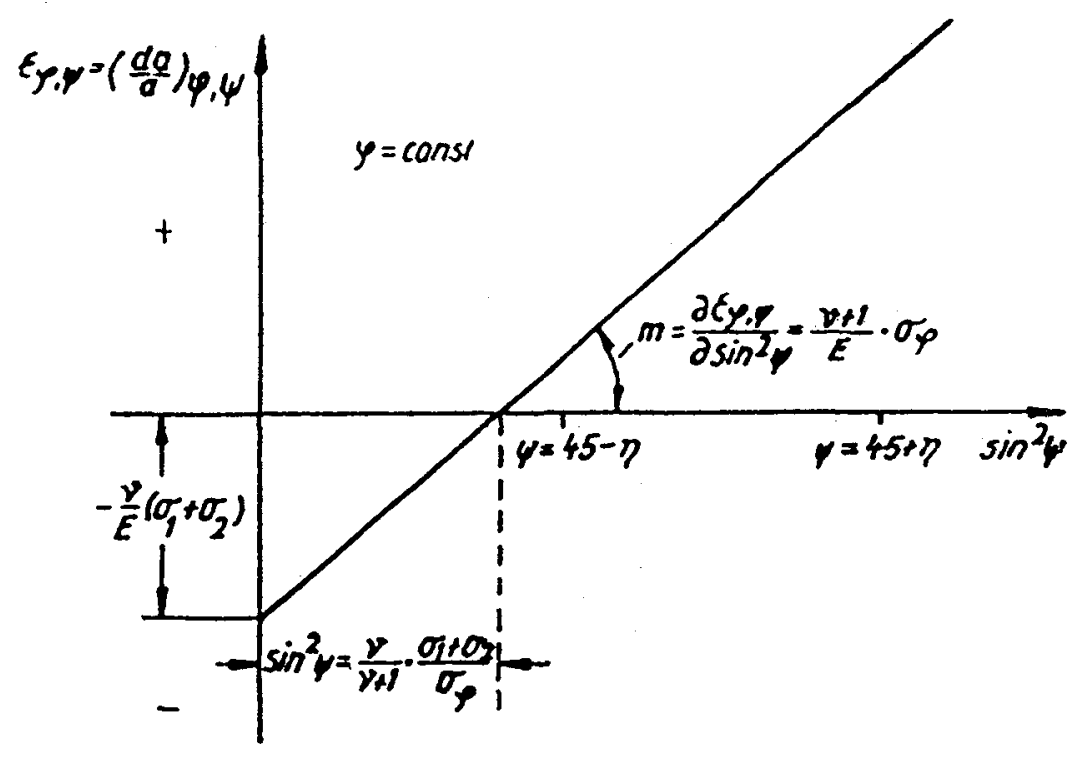

Fig. 14 : Evolution des déformations de réseau en fonction de $\sin ^{2} \psi$ dans l'azimut $\varphi=$ const. pour un état de contrainte blaxial. 
Macherauch et Müller établirent en plus une méthode précise et fiable pour déterminer les constantes élastiques radiocristallographiques [54], qui permit dans les années suivantes d'abandonner la mesure de l'anisotropie $\xi$. Les incertitudes relatives à l'influence de l'anisotropie élastique purent être levées en appliquant la méthode ci-dessus pour la détermination des constantes élastiques radiocristallographiques par rayons $\mathrm{X}$. On réalise des essais de traction ou de flexion sous différentes contraintes extérieures afin d'obtenir les constantes élastiques radiocristallographiques dans un matériau pour les plans $\{\mathrm{hkl}\}$ considérés. Les variations de déformation du réseau résultantes des variations des contraintes externes sont mesurées pour différents angles $\psi$ et tracées en fonction de $\sin ^{2} \psi$. En raison des relations théoriques d'élasticité, on s'attend à ce que les points de mesure forment une droite (figure 15). La pente de la droite permet d'obtenir $1 / 2 S_{2}$ et l'ordonnée à l'origine fournit $S_{1}$.

Le développement de la méthode d'obtention des contraintes cristallographiques s'inspira essentiellement d'une idée de Wolfstieg [55] en 1959. Ici, le principe n'est plus d'incliner la surface de l'échantillon de l'angle $\psi$ autour de l'axe $2 \theta$. On définit plutôt un nouvel axe $\psi$, perpendiculaire à l'axe $2 \theta$ du diffractomètre, et déterminé par l'intersection du plan du diffractomètre et de la surface de l'échantillon (figure 13b). De tels diffractomètres $\Psi$ présenteraient un avantage pratique notable par rapport aux diffractomètres $\Omega$ utilisés jusqu'alors.

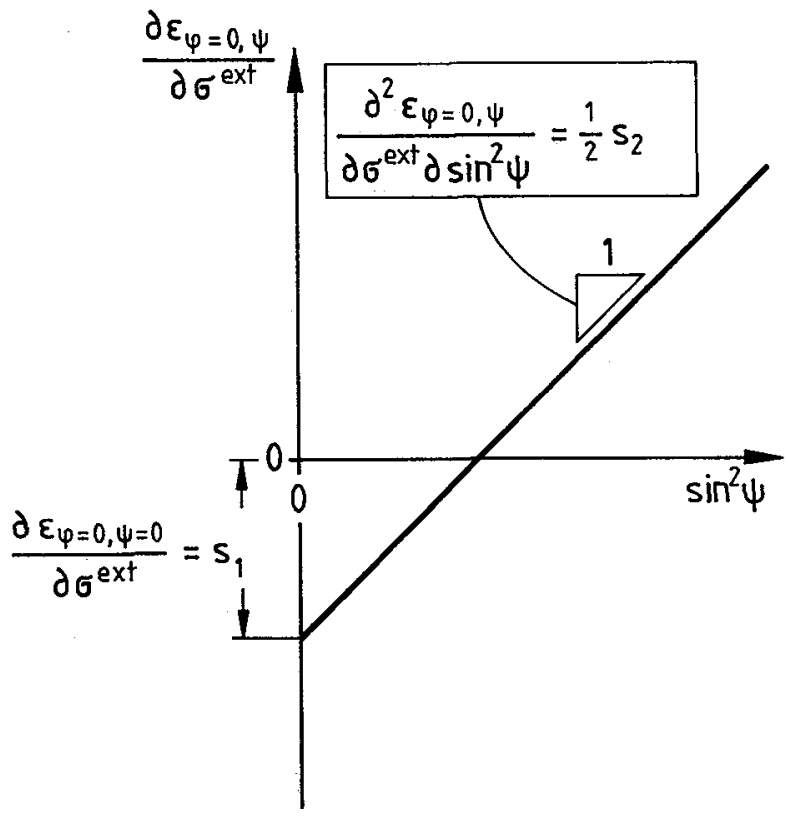

Fig. 15 : Evolution attendue de la variation des déformations du réseau à la suite de variation des contraintes externes en fonction de $\sin ^{2} \psi$ pour la détermination expérimentale des constantes élastiques radiocristallographiques (CER).

Les développements ci-dessus conduisirent à un progrès remarquable dans la technique de mesure des contraintes par rayons $\mathrm{X}$, qui étaient jusque là imprégnées de nombreuses incertitudes. Des groupes de travail furent formés en 1960 en Allemagne, en 1962 au Japon. Les scientifiques et ingénieurs y échangeaient régulièrement leurs connaissances dans le domaine de l'analyse des contraintes résiduelles. Cela favorisa l'échange des connaissances 
entre les groupes de chercheurs allemands et japonais [56]. Dans les années suivantes, les techniques de mesure de contraintes connurent un élargissement remarquable. Il en résulta de nombreuses recherches et développements concernant d'une part la détermination et l'exploitation des contraintes issues de déformations du réseau, et d'autre part, les appareillages. Ainsi, Neff [57] en 1963 et aussi Kolb et Macherauch [58] en 1964 développèrent les premiers diffractomètres mobiles à l'aide desquels des analyses de contraintes par rayons $\mathrm{X}$ purent être réalisées au dehors de laboratoire directement sur des éléments de constructions, éventuellement de taille imposante.

En 1967, Bollenrath et col. [59] appliquèrent le modèle de couplage des cristallites de Kröner pour calculer les constantes élastiques radiocristallographiques. Dans ce modèle, chaque cristallite est considéré comme une inclusion élastique anisotrope dans une matrice isotrope. Des études furent faites avec un rayonnement $\mathrm{Cu} \mathrm{K} \alpha$ pour déterminer les constantes radiocristallographiques sur les plans $\{420\}$ d'alliages $\mathrm{Cu}-\mathrm{Ni}$ de différentes compositions [60] (figure 16). Les résultats mesurés sont bien en accord avec l'évolution calculée sous l'hypothèse de Kröner et avec les valeurs moyennes obtenues par les modèles de Voigt et Reuss.
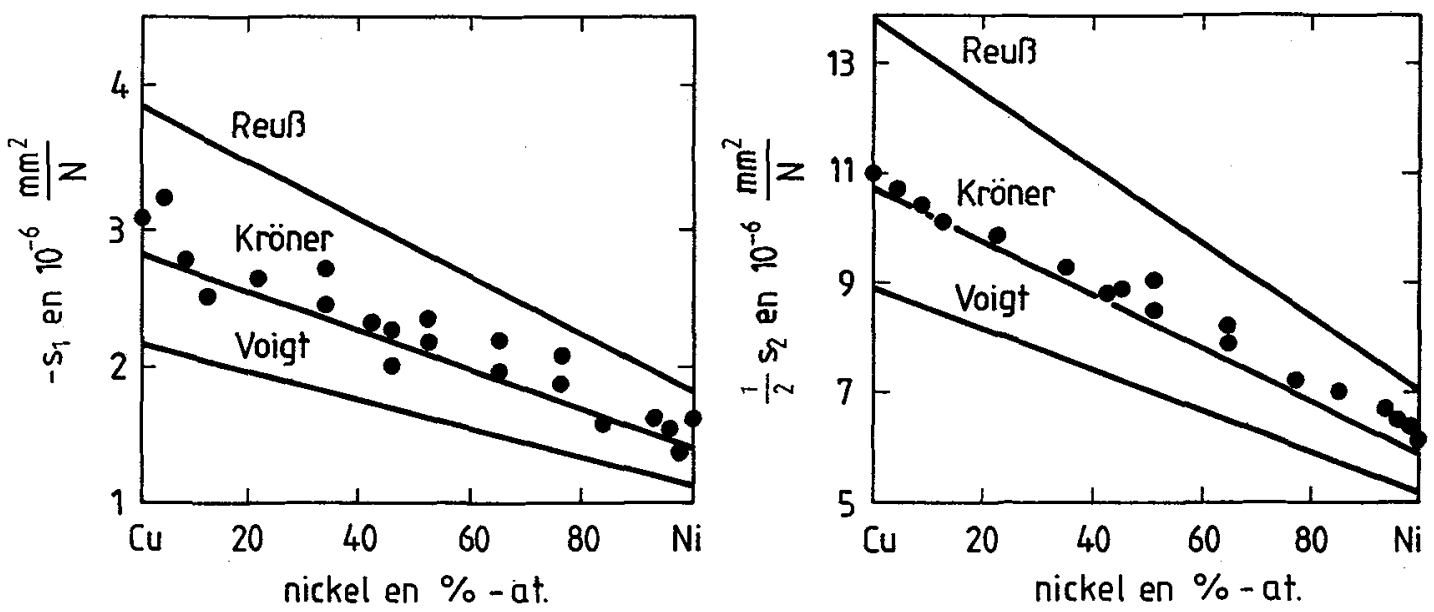

Fig. 16 : Constantes élastiques radiocristallographiques expérimentales obtenues par rayonnement $\mathrm{Cu} \mathrm{Ka}$ sur les plans $\{420\}$ et valeurs théoriques calculées selon Voigt, Reuss et Kröner pour des alliages cuivre-nickel en fonction de la teneur en nickel.

Dès 1967, Shiraiwa et Sakamoto discutèrent de l'influence de la profondeur de pénétration du rayonnement $X$ en présence de gradients de contraintes résiduelles proche de la surface dans des aciers durs [61]. En 1967 et 1968, Taira et col. déterminèrent les constantes élastiques radiocristallographiques de matériaux texturés en fonction du degré de déformation $[62,63]$. En 1972, Kelly et Eichen utilisèrent pour la première fois un diffractomètre piloté par ordinateur [64].

Déjà en 1965 , Fuchs et Gladkikh avaient observé de manière systématique les déformations de réseau différentes dans des aciers pour des même angles $\psi$ positifs et négatifs [65]. Ces résultats furent confirmés en 1973 par Diament [66] et Walburger [67] sur des aciers polis. Pour expliquer cela, Evenschor et Hauk [68], ainsi que Christ et Krause [69] se sont basés sur l'équation (14) établie en 1937 par Romberg, afin de calculer les contraintes à partir de la 
courbe de déformation en fonction de $\sin ^{2} \psi$ et ont obtenu une ellipse. Cette équation peut s'écrire

$$
\varepsilon_{\varphi, \psi}=\mathrm{A} \sin ^{2} \psi+\mathrm{B} \sin (2 \psi)+C .
$$

A partir de là, Dölle et Hauk développèrent en 1976 un procédé pour déterminer les états de contrainte triaxiaux avec une orientation quelconque des axes principaux [70]. En faisant la moyenne et la différence des déformations du réseau pour des angles $\psi$ positifs et négatifs, ils obtinrent à partir de l'équation (16)

et

$$
a_{\psi}^{+}=\frac{1}{2}\left(\varepsilon_{\varphi, \psi^{+}}+\varepsilon_{\varphi, \psi}-\right)=A \sin ^{2} \psi+C
$$

$$
\mathrm{a}_{\psi}^{-}=\frac{1}{2}\left(\varepsilon_{\varphi, \psi^{+}}-\varepsilon_{\varphi, \psi^{-}}\right)=\mathrm{B} \sin 2 \psi
$$

On obtient ainsi les équations des droites en fonction de $\sin ^{2} \psi$ et $\sin (2 \psi)$. Les pentes de ces droites, représentées sur la figure 17 , permettent d'obtenir les composantes des contraintes $\mathrm{A}=$ $1 / 2 S_{2} \sigma_{\varphi}$, et $\mathrm{B}=1 / 2 \mathrm{~S}_{2}\left(\sigma_{13} \cos \varphi+\sigma_{23} \sin \varphi\right)$. L'évolution ellipsoïdale des déformations du réseau en fonction de $\sin ^{2} \psi$ s'explique donc par les contraintes de cisaillement correspondantes $\sigma_{13}$ et $\sigma_{23}$. En 1977, Wakabayashi et col. [71] montrèrent l'augmentation de la largeur de l'ellipse avec le taux de carbone des aciers polis (figure 18). Les mesures pour le fer Armco ne présentent pas d'ellipse.

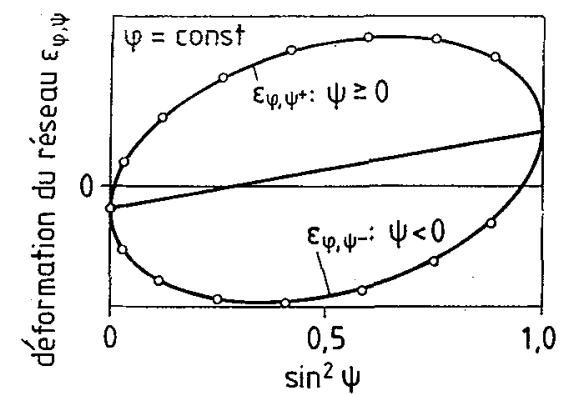

$$
a_{\psi}^{+}=\frac{1}{2}\left(\varepsilon_{\varphi, \psi^{+}}+\varepsilon_{\varphi, \psi^{-}}\right)
$$
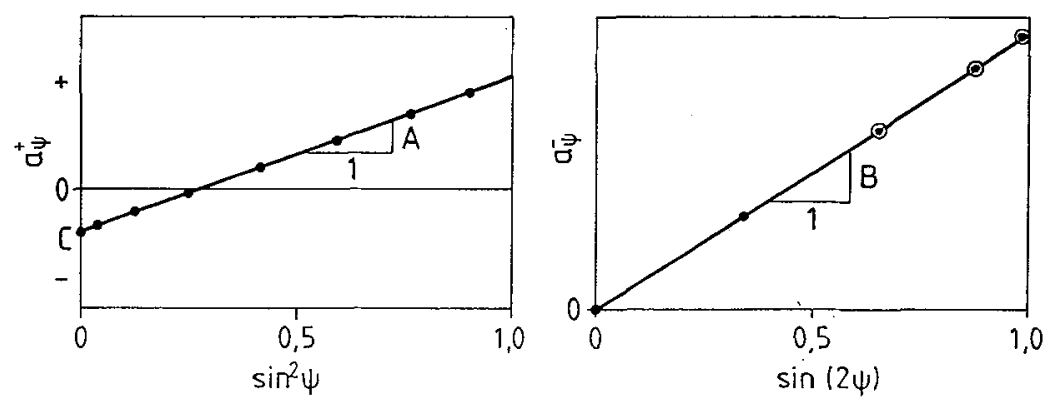

Fig. 17 : Evaluation de l'évolution ellipsoïdale des déformations du réseau en fonction de $\sin ^{2} \psi \mathrm{d}$ 'après Dölle et Hauk. 
En 1976, Barett et Predecki [72] analysèrent les déformations de réseau par rayons X de polymères auxquels ils mélangèrent de la poudre cristalline. La même année, Nagao et Kusumoto [73] firent des analyses de contraintes en diffraction en énergie avec un rayonnement polychromatique en utilisant un détecteur à haute résolution. James et Cohen employèrent un détecteur à localisation linéaire pour l'analyse des contraintes par rayons $\mathrm{X}$ [74].
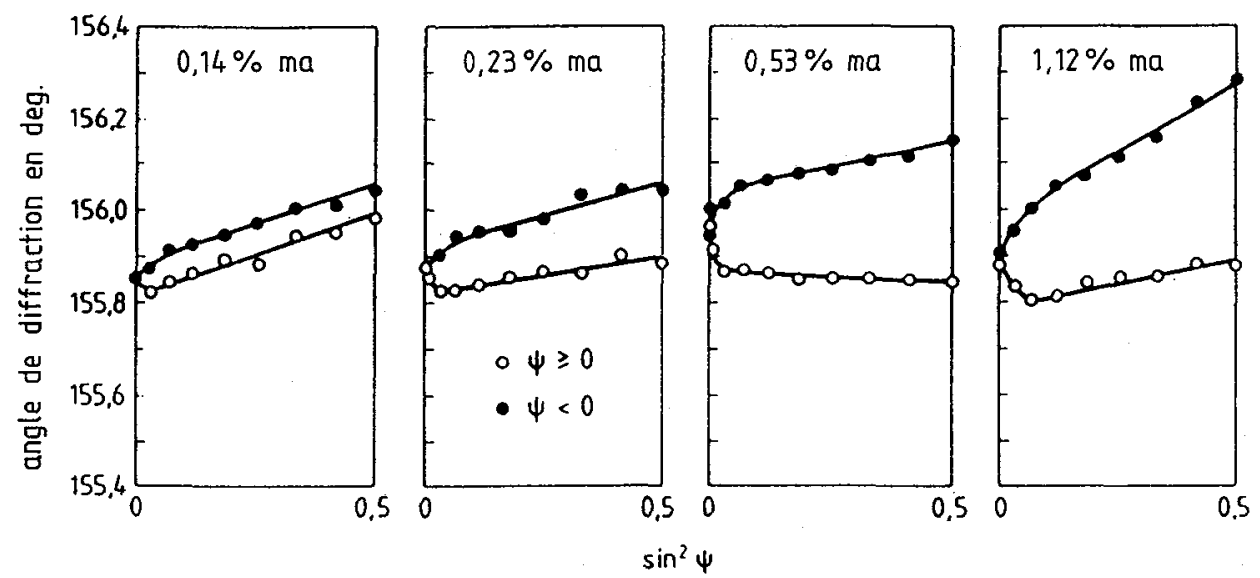

Fig. 18 : Evolutions ellipsoïdales des angles de diffraction en fonction de $\sin ^{2} \psi$ pour des aciers polis avec différentes concentrations en carbone.

\section{DEVELOPPEMENT DE L'ANALYSE DES CONTRAINTES PAR RAYONS $X$ APRES 1980 ET ETAT ACTUEL}

A partir du début des années 1980, l'analyse des contraintes par rayons X se développa d'autant plus que grandit l'intérêt mondial pour les contraintes résiduelles, leur naissance, leurs mesures, leur calcul, leurs effets sur les éléments de construction. Ceci découla logiquement du fait que dans de nombreux cas, des améliorations importantes des propriétés des éléments de construction pouvaient être obtenues en présence de certaines contraintes résiduelles lors de sollicitations mécaniques et/ou corrosives. Puis, on se rendit compte qu'en pratique les éléments de construction présentent presque toujours des contraintes résiduelles macroscopiques et toujours des contraintes résiduelles microscopiques. Les développements qui en résultèrent au niveau de l'analyse des contraintes par rayons $\mathrm{X}$ répondirent à de nombreuses questions datant de plusieurs dizaines d'années, ceci à l'aide de meilleurs appareils et de connaissances scientifiques fortement élargies. D'autre part, depuis le début des années 1980, un développement sans précédent eut lieu dans le domaine des sciences et techniques des matériaux. Dans le domaine des céramiques, des matériaux composites, et dans la technologie des dépôts de couche(s), celui-ci provoqua le développement de nouveaux matériaux tels que les phases intermétalliques, ainsi que des développements dans la microélectronique et dans la technique des microsystèmes. Ceci fut, et est encore, à l'origine d'une multitude de procédés scientifiques pour l'analyse des contraintes par rayons $\mathrm{X}$.

En 1984, Barral et col. utilisèrent pour la première fois dans l'histoire de l'analyse des contraintes un rayonnement $X$ issu d'un synchrotron [75]: La forte intensité et le parallélisme de ces rayons permirent une excellente résolution à la surface de l'échantillon et à une distance 
par rapport à la surface de l'ordre de grandeur de la profondeur de pénétration. En 1985, Crostack et col. présentèrent un procédé pour l'analyse des contraintes par rayons X sur des gros cristallites individuelles des polycristaux [76]. Ils le mirent en application avec un rayonnement synchrotron pour l'analyse des contraintes résiduelles et externes sur des alliages à base de nickel. En 1988, Hauk et col. [77] contribuèrent à l'explication de l'influence de l'anisotropie élastique des matériaux texturés sur les résultats de l'analyse des contraintes. Le procédé de groupes de cristallites qu'ils développèrent présuppose que tous les cristallites de ce groupe sont orientés idéalement par rapport à la direction de texture considérée. Ceci permet la détermination des contraintes résiduelles dans ce groupe de cristallites, en tenant compte de l'anisotropie élastique du cristallite ainsi que du modèle de couplage de Reuss.

Finalement, en se fondant sur une idée de Peiter et Lode [78], Eigenmann et col. appliquèrent le procédé de l'intégrale $\varphi$ à l'analyse des contraintes résiduelles dans des couches PVD minces de $\mathrm{TiC}$ avec des textures de fibres [79]. Pour ce procédé, l'équation (14) peut être écrite sous la forme d'une série de Fourrier en $\varphi$

$$
\varepsilon_{\varphi, \psi}=\frac{1}{2} A_{0}+A_{1} \cos \varphi+A_{2} \cos (2 \varphi)+B_{1} \sin \varphi+B_{2} \sin (2 \varphi)
$$

Les coefficients de Fourrier $A_{n}$ et $B_{n}$ sont fonctions de $\psi$ et des composantes du tenseur des contraintes $\left(\sigma_{i j}\right)$ que l'on cherche à déterminer. On a

$$
\begin{aligned}
& \frac{1}{2} A_{o}=\frac{1}{2} S_{2}\left[\frac{1}{2}\left(\sigma_{11}+\sigma_{22}\right)-\sigma_{33}\right] \sin ^{2} \psi+\frac{1}{2} S_{2} \sigma_{33}+S_{1}\left(\sigma_{11}+\sigma_{22}+\sigma_{33}\right) \\
& A_{1}=\frac{1}{2} S_{2} \sigma_{13} \sin (2 \psi), \quad A_{2}=\frac{1}{2} S_{2} \frac{1}{2}\left(\sigma_{11}-\sigma_{22}\right) \sin ^{2} \psi, \\
& B_{1}=\frac{1}{2} S_{2} \sigma_{23} \sin (2 \psi) \quad \text { et } \quad B_{2}=\frac{1}{2} S_{2} \sigma_{12} \sin ^{2} \psi
\end{aligned}
$$

Pour les valeurs de $\psi$ où apparaissent les pôles de la texture de fibre, on mesure un nombre suffisamment grand de valeurs de déformation de réseau $\varepsilon_{\varphi, \psi}$ en fonction de $\psi$. Les coefficients de Fourrier sont obtenus par intégration numérique

$$
A_{n}=\frac{1}{\pi} \int_{0}^{2 \pi} \varepsilon_{\varphi, \psi} \cos (n \varphi) d \varphi . \quad(21) \quad \text { et } \quad B_{n}=\frac{1}{\pi} \int_{0}^{2 \pi} \varepsilon_{\varphi, \psi} \sin (n \varphi) d \varphi .
$$

Les contraintes ainsi obtenues pour des couches PVD en TiC de $5 \mu \mathrm{m}$ sur le métal dur WC-Co et les constantes de réseau pour un état sans contrainte sont présentées sur la figure 19 en fonction de la pression du procédé lors du dépôt de la couche. Pour déterminer ces constantes 
de réseau, on suppose un état de contraintes résiduelles biaxiales dans les couches, suivant la méthode proposée par Torbaty et col. [80]. A l'évidence, les contraintes résiduelles de pression et les constantes de réseau des couches décroissent fortement avec une pression croissante du procédé. Lorsque la pression augmente, l'énergie des particules gazeuses diminue et ceci a pour conséquence une diminution du nombre d'atomes de carbone dans les sites interstitiels du réseau de la couche.
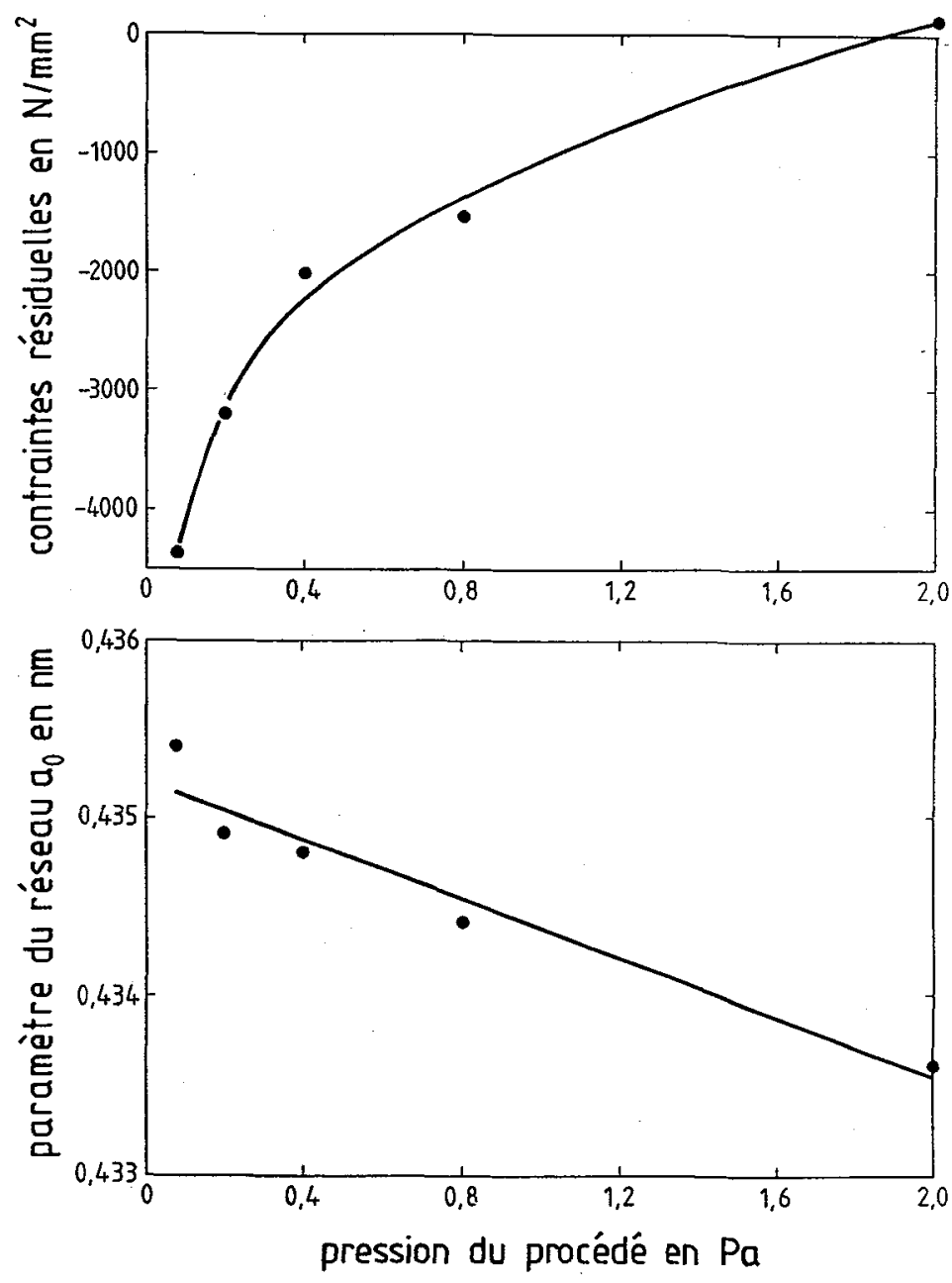

Fig. 19 : Contraintes résiduelles (a) et paramètres de réseau (b) des couches PVD de TiC de $5 \mu m$ d'épaisseur en fonction de la pression du procédé lors du dépot de la couche.

Au début des années 90 , la discussion au sujet des procédés dérivés de l'équation (14) pour analyser les états de contraintes homogènes d'un matériau isotrope au niveau macroscopique était réglée. Au moment de la genèse de l'analyse des contraintes par rayons $\mathrm{X}$, alors que l'exploitation des mesures se faisait graphiquement, il était important de reporter linéairement 
les contraintes mesurées. Cette exigence conduisit d'abord au procédé en $\sin ^{2} \psi[53]$ et, plus tard, au procédé de Dölle et Hauk [70]. Ces procédés sont encore utilisés aujourd'hui pour expliquer concrètement l'analyse des contraintes par rayons X. Jusqu'à la fin des années 80 , la limitation des capacités des ordinateurs conduit à des temps de calculs importants. C'est pourquoi il était préférable de découpler le système de 6 équations à 6 inconnues en sous systèmes de plus petite dimension pour simplifier le calcul des contraintes. Ceci a été réalisé par l'application du procédé Dölle et Hauk ou de l'intégrale $\varphi$. Aujourd'hui, la validité et la comparaison de tous ces procédés et leurs avantages sont reconnus. Pour le calcul des contraintes à l'aide des mesures des déformations du réseau, il est aujourd'hui néanmoins plus adéquat de résoudre l'équation (14) dans le cas le plus général ou simplifiée pour certains états donnés suivant l'un des algorithmes usuels. Pour cela, si l'on suppose $\sigma_{33}=0$, on peut introduire la position $2 \theta_{0}$ des raies de diffraction du réseau libre de contraintes comme inconnue dans le système d'équations.

Du point de vue de la théorie de l'élasticité, on ajoute également à la solution la plus générale le problème de l'analyse des contraintes par rayons X pour des matériaux texturés. En 1983, puis 1987, Barral et col. [81, 82] ainsi que Serruys et col. [83], publièrent des méthodes dans lesquelles la fonction de distribution des orientations (FDO) caractérise la texture et sert aussi à l'exploitation des mesures de déformation de réseau par rayons $\mathrm{X}$. On obtient la relation

$$
\varepsilon_{\varphi, \psi}=\mathrm{F}_{\mathrm{ij}}\left(\varphi, \psi, \mathrm{FDO}, \mathrm{s}_{\mathrm{ijkl}}\right) \sigma_{\mathrm{ij}} .
$$

qui permet, à l'aide de la fonction de la distribution des orientations, de déterminer les composantes des contraintes intéressantes, à condition d'effectuer suffisamment de mesures de $\varepsilon_{\varphi, \psi}$ dans différentes directions $\varphi, \psi$. Le modèle de couplage de cristallites de Reuss (dans lequel les contraintes sont homogènes) est à l'origine de cette méthode $[81,82]$. Au contraire [83], on montre qu'avec cette hypothèse, les effets de l'anisotropie élastique sont surévalués. Ceci est montré sur la figure $20 \mathrm{a}$ où les distances interréticulaires entre des plans de réseau $\{211\}$ d'un acier laminé à froid sont représentées en fonction de $\sin ^{2} \psi$. Si on suppose que le modèle de couplage des cristallites de Voigt (déformations homogènes dans chaque cristallite) est vrai, alors on obtient une régression linéaire à partir des points expérimentaux (figure 20b). En combinant les deux méthodes, on peut optimiser la correspondance des points expérimentaux avec la courbe de régression (figure 20c). En fait, il y a alors deux contributions à la non-linéarité de la courbe. La première est due à l'anisotropie élastique liée à la texture présente. La deuxième contribution résulte des contraintes résiduelles de type II dues aux différentes déformations plastiques dans des cristallites de différentes orientations. Seule l'anisotropie élastique peut être interprétée de manière correcte par les relations de la théorie élastique décrite ci-dessus.

En 1987 Hauk et col. publièrent un rapport intéressant sur la détermination des constantes élastiques radiocristallographiques et sur l'évolution des contraintes résiduelles dans l'épaisseur de la paroi d'un cylindre en polypropylène extrudé [84]. Des mesures effectuées avec un rayonnement respectivement $\mathrm{Cr} K \alpha$ et $\mathrm{Cu} K \alpha$ sur les plans $\{130\}$ des domaines cristallins du matériau semi-cristallin permirent de déterminer les distances entre les plans de réseau en réflexion comme en transmission pour des angles de diffraction respectivement $2 \theta \approx$ $27,5^{\circ}$ et $2 \theta \approx 18,5^{\circ}$. Ces distances entre plans sont tracées en fonction de $\sin ^{2} \psi$ sur la figure 21. On a réalisé plusieurs mesures de contraintes en réflexion et en rabotant peu à peu des couches de matériau. Les résultats, après mesure et après correction, sont tracés en fonction de l'épaisseur de la paroi sur la figure 22a. L'évolution des contraintes ainsi obtenues dans 
l'épaisseur de la paroi du cylindre concorde bien avec les résultats obtenus en transmission sans rabotage (figure $22 \mathrm{~b}$ ).

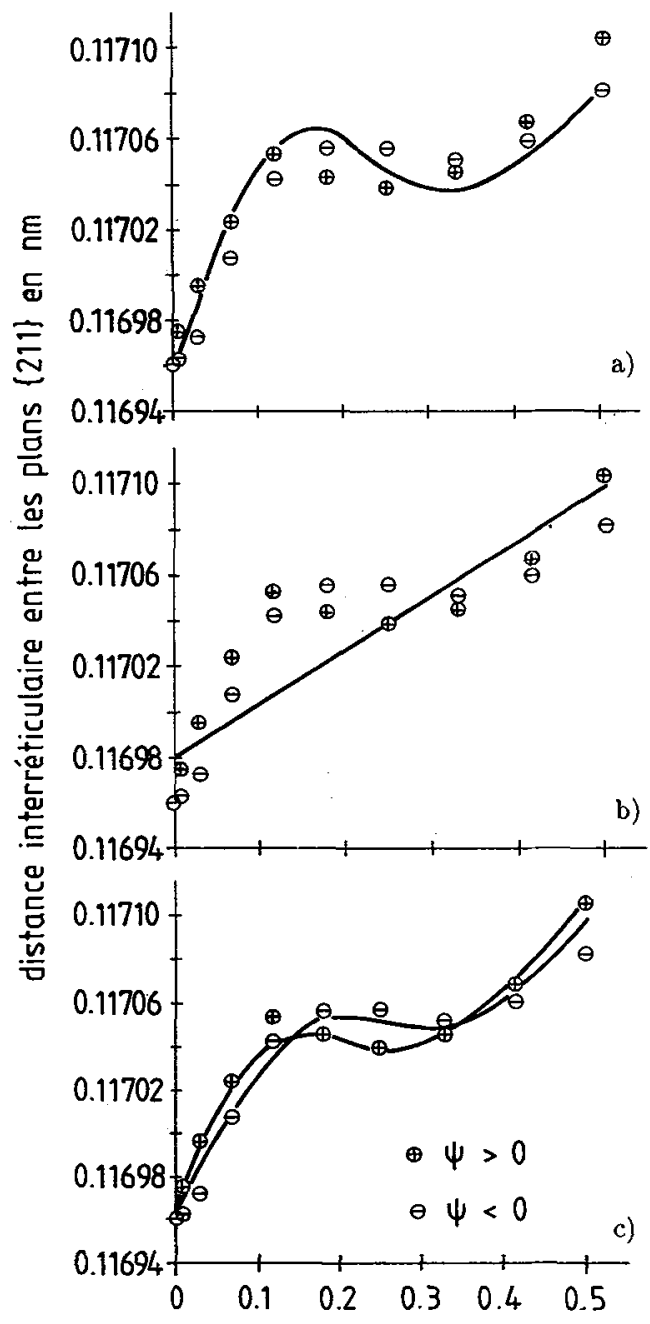

Fig. 20 : Distances interréticulaires entre les plans $\{221\}$ de la ferrite d'un acier laminé à froid déterminées par rayonnement $\mathrm{Cr} \mathrm{K} \alpha$ en fonction de $\sin ^{2} \psi$ ainsi que les courbes de régression calculées en tenant compte de la fonction de distribution des orientations avec le modèle de couplage des cristallites de Reuss (a), de Voigt (b) et avec un modèle intermédiaire (c). 

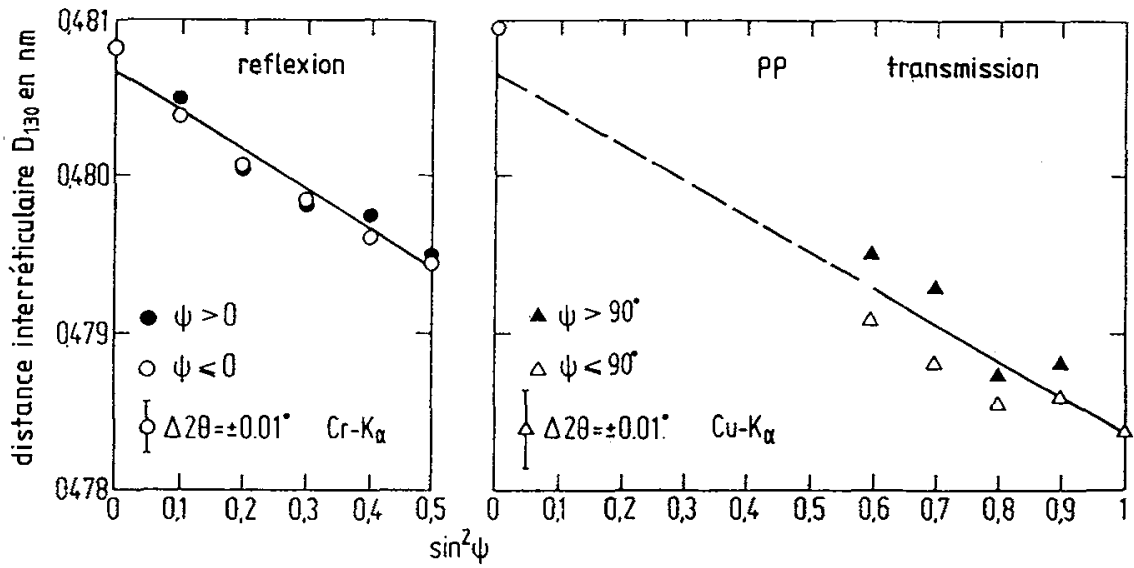

Fig. 21 : Distances interréticulaires entre les plans $\{130\}$ du réseaux de polypropylène, mesurées en fonction de $\sin ^{2} \psi$ par réflexion d'un rayonnement $\mathrm{Cr} \mathrm{K} \alpha$ (a) et par transmission d'un rayonnement $\mathrm{Cu} \mathrm{K} \alpha$ (b).

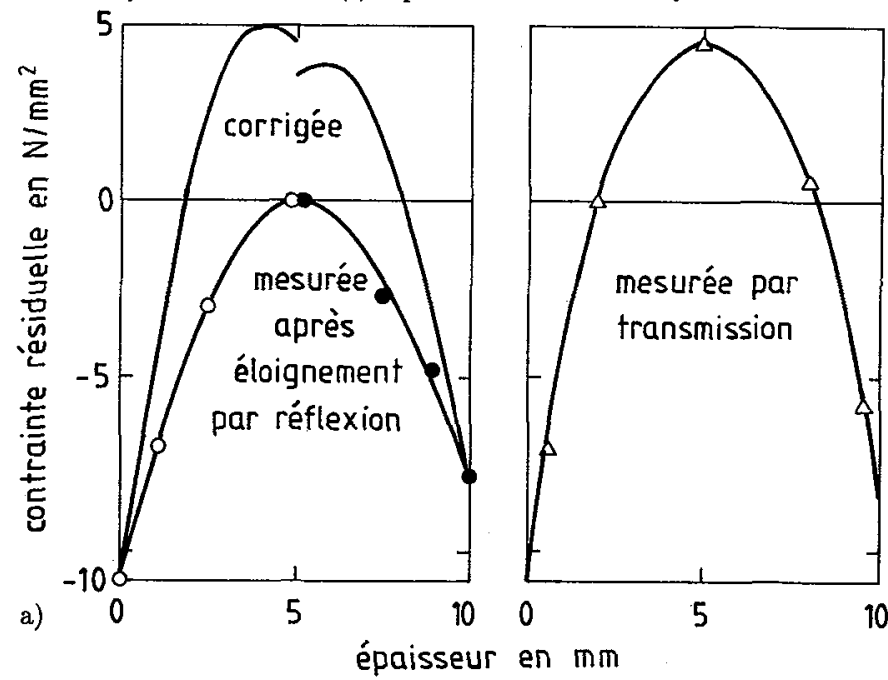

b)

Fig. 22 : Evolution des contraintes résiduelles en fonction de l'épaisseur de la paroi d'un tube de polypropylène extrudé, obtenue par réflexion (a) et en transmission (b).

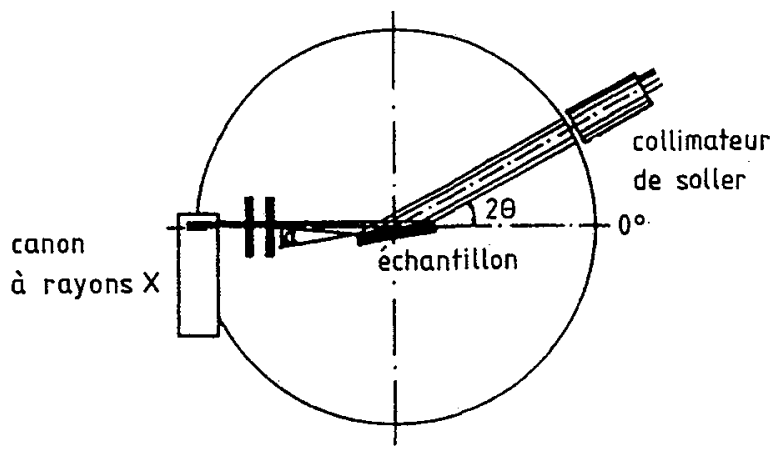

Fig. 23 : Montage pour déterminer les contraintes dans des couches minces par diffraction sous des angles d'incidence $\alpha$ faibles. 
Pour l'analyse des contraintes par rayons $\mathrm{X}$ dans des couches d'épaisseurs inférieures à la profondeur de pénétration du rayonnement $X$ dans la couche, le problème principal est que la partie du rayonnement diffractée par la couche est très faible. Les raies de diffraction sont donc difficilement, voire impossible à interpréter. Pour diminuer la profondeur de pénétration du rayonnement, il faut réduire l'angle $\alpha$ d'incidence des rayons $\mathrm{X}$ à quelques degrés. De Buyser et col. [85] déterminèrent en 1990 les déformations de réseau pour différents plans $\{$ hkl $\}$ aves la configuration de la figure 23. Les différentes positions des raies $2 \theta$ entraînent différents angles $\psi=\theta-\alpha$. La figure 24 montre les déformations du réseau en fonction de $\cos ^{2} \psi$ pour une couche de nickel de $2 \mu \mathrm{m}$ sur du cuivre mesurées avec $\alpha=6^{\circ}$ et un rayonnement $\mathrm{Cu} \mathrm{Ka}$. En utilisant cette courbe et le modèle de couplage de Voigt, on obtient dans la couche des contraintes résiduelles de $363 \mathrm{MPa}$, avec le modèle de Reuss $332 \mathrm{MPa}$. Les auteurs soulignent que pour de faibles angles $\alpha$ d'incidence de petites erreurs de positionnement de l'échantillon ont une très grande influence sur les résultats de mesure. Il est donc nécessaire de poursuivre les recherches pour pouvoir déterminer les contraintes résiduelles dans les couches et réduire ces effets en travaillant avec des rayons parallèles.

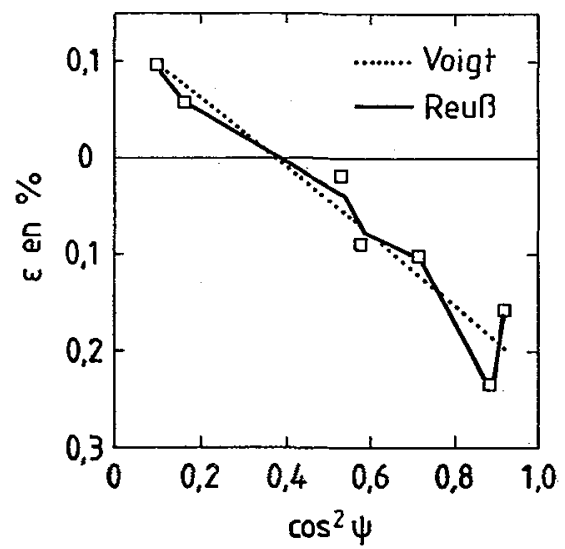

Fig. 24 : Evolution des déformations du réseau en fonction de $\cos ^{2} \psi$ d'une couche de nickel de $2 \mu \mathrm{m}$ d'épaisseur sur du cuivre.

Dans les années 1995 et 1996, des progrès notables furent faits pour déterminer les états de contraintes résiduelles ayant des gradients près de la surface, comme c'est souvent le cas pour des céramiques polies. Pour cela, on se basa sur les travaux de Ruppersberg et col. [86]. Le problème principal réside dans le fait que les déformations de réseau mesurées

$$
\varepsilon_{\varphi, \psi}=\frac{\int_{0}^{s} \varepsilon_{\varphi, \psi}(z) e^{-z / z^{*}} d z}{\int_{0}^{S} e^{-z / z^{*}} d z}
$$

sont des valeurs moyennes des déformations en profondeur $\varepsilon_{\varphi, \psi}(\mathrm{z})$ rapportées par la fonction d'affaiblissement des rayons $\mathrm{X}$ dans le matériaux. A la suite de cela, les valeurs des contraintes résiduelles déterminées par les valeurs $\varepsilon_{\varphi, \psi}$ mesurées sont données par les valeurs moyennes 


$$
\sigma_{i j}=\frac{\int_{0}^{S} \sigma_{i j} e^{-z / z^{*}} d z}{\int_{0}^{S} e^{-z / z^{*}} d z}
$$

des contraintes en profondeur $\sigma_{\mathrm{ij}}(\mathrm{z})$. Comme l'épaisseur de l'échantillon peut, la plupart du temps, être considérée comme infinie, la détermination de l'évolution des contraintes résiduelles nécessite l'inversion de l'intégrale de Laplace. Ce problème étant particulièrement mal conditionné, il faut mesurer des déformations de réseau pour des angles $\psi$ allant jusqu'à près de $90^{\circ}$. La figure $25 \mathrm{a}$ montre l'évolution de la position des raies diffractées $2 \theta_{\varphi, \psi}$ par rapport à $\sin ^{2} \psi$ pour une céramique polie qui a été mesurée avec un rayonnement synchrotron dans l'intervalle $-89^{\circ}<\psi<89^{\circ}$ [87]. La figure $25 \mathrm{~b}$ présente les évolutions en profondeur des contraintes résiduelles près de la surface obtenues par le procédé polynômial [88]. Pour ce procédé, on utilise des polynômes pour interpréter les évolutions en profondeur des contraintes résiduelles et on choisit un degré favorable. Leverenz et col. divisèrent l'épaisseur de l'échantillon en petits intervalles, ce qui permet de définir pour chaque intervalle un polynôme de degré inférieur (figure 26), la continuité et la dérivabilité aux limites de deux intervalles doivent toujours rester vraies. Une telle méthode permet encore d'améliorer la stabilité de la solution [89].
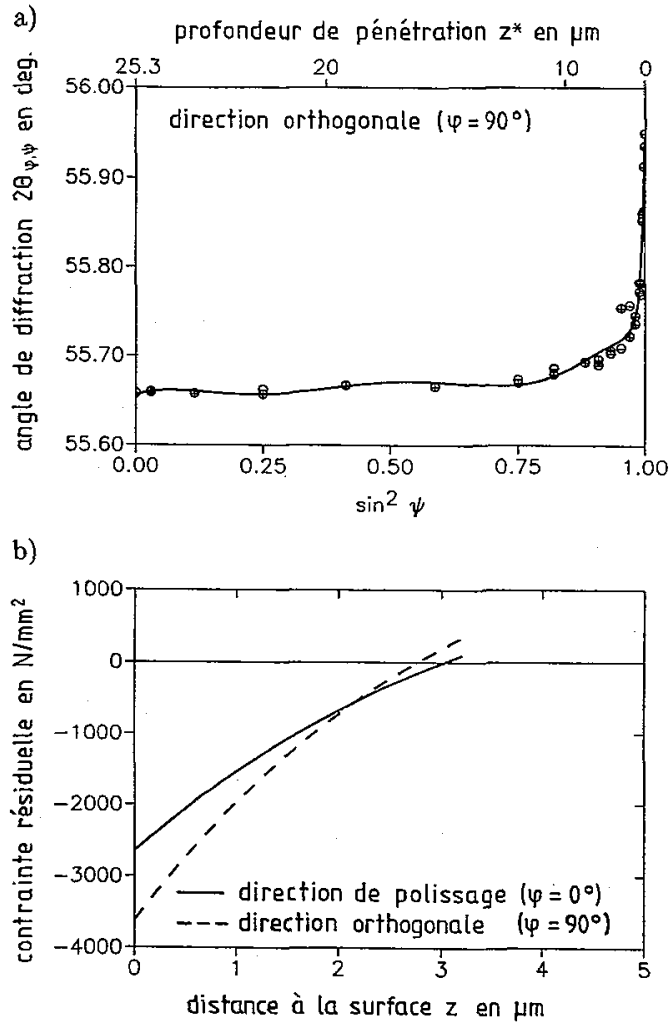

Fig. 25 : Angles de diffraction $2 \theta_{\varphi, \psi}$ d'une céramique $\mathrm{Al}_{3} \mathrm{O}_{3} / \mathrm{TiC}$ (5\% Vol.) mesurées avec un rayonnement synchrotron en fonction de $\sin ^{2} \psi$ (a) et évolution en profondeur des contraintes résiduelles près de la surface évaluée à l'aide du procédé polynômial (b). 
Depuis le début des années 80 , les connaissances dans le domaine de l'analyse des contraintes par rayons $\mathrm{X}$ et ses applications sont échangées régulièrement dans le cadre de conférences internationales (ICRS) et européennes (ECRS), les exposés lors de ces conférences sont consignés dans un livre. En 1983 eut lieu à Karlsruhe la première conférence européenne sur les contraintes résiduelles. En 1986 à Garmisch-Partenkirchen, en Allemagne, se tint la première conférence internationale. Les dates des rencontres sont listées sur la figure 27 . La prochaine conférence européenne aura lieu en 1996 à Cluny en France et la prochaine conférence internationale en 1997 à Linköping en Suède.

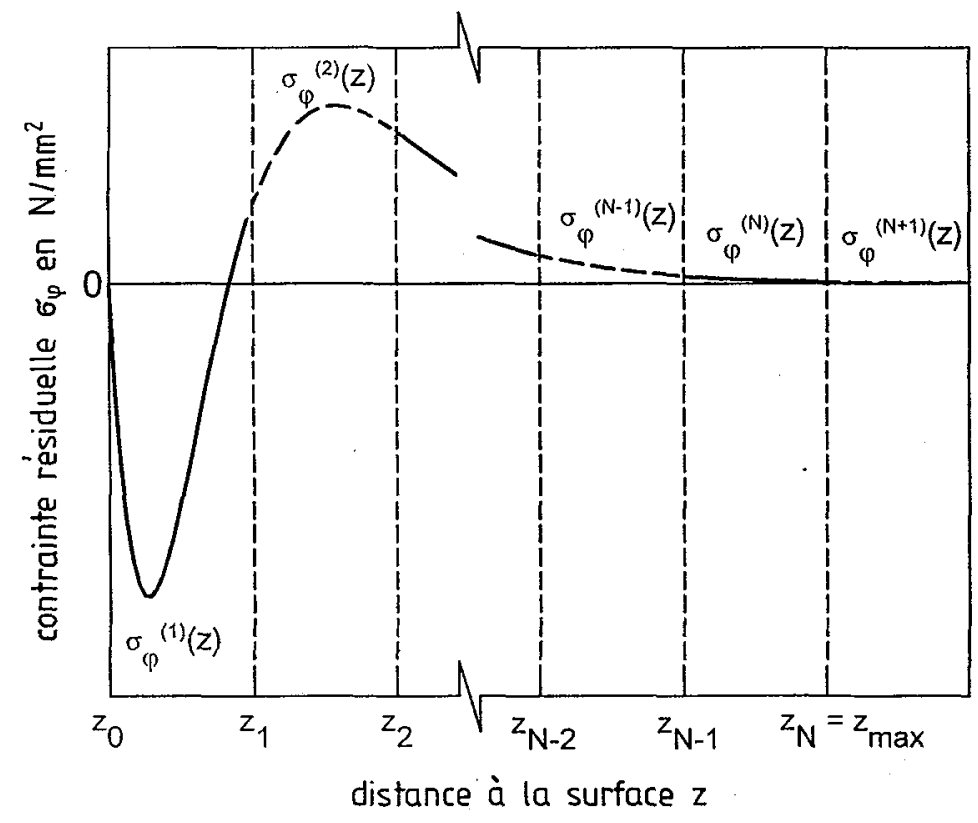

Fig. 26 : Représentation schématique de l'évolution d'une contrainte résiduelle par fonctions définies dans différents intervalles d'après Leverenz et d'autres.

\begin{tabular}{|c|c|c|}
\hline & ICRS & ECRS \\
\hline 1983 & & Karlsruhe / Allemagne \\
\hline 1986 & $\begin{array}{l}\text { Garmisch-Partenkirchen } \\
\text { Allemagne }\end{array}$ & \\
\hline 1988 & Nancy / France & \\
\hline 1990 & & Darmstadt / Allemagne \\
\hline 1991 & Tokushima / Japon & \\
\hline 1992 & & Frankfurt / Allemagne \\
\hline 1994 & Baltimore / États Unis & \\
\hline 1996 & & Cluny / France \\
\hline 1997 & Linköping / Suède & \\
\hline
\end{tabular}

Fig. 27 : Calendrier des conférences internationales (ICRS) et européennes (ECRS) sur le thème des contraintes résiduelles. 


\section{CONCLUSIONS}

Cet historique montre clairement que l'analyse des contraintes par rayons $\mathrm{X}$ a connu depuis ses débuts (années 20) une formidable évolution et un remarquable élargissement. Aujourd'hui, de nombreux problèmes spécifiques à cette méthode ont été compris et résolus. En plus des méthodes standards pour l'analyse des états de contraintes homogènes dans des matériaux isotropes au niveau macroscopique, on a développé de nombreuses variantes de méthodes pour l'analyse de contraintes dans des cristallites individuels de polycristaux, pour l'analyse de contraintes résiduelles dans des couches très minces, en présence de texture et de gradients de contraintes résiduelles. L'avancée du développement dans le domaine des sciences des matériaux et de l'ingénieur, notamment dans la fabrication et l'application de nouveaux composites et matériaux à couches, et dans les techniques des microsystèmes soulèvent toujours des problèmes en ce qui concerne les techniques de mesure et l'élaboration des matériaux pour les contraintes résiduelles. Ceci, et les développements très prometteurs dans le conditionnement du rayonnement (réalisé soit grâce aux miroirs à couches multiples, soit grâce aux capillaires en verre focalisant rendant le faisceau de rayons $\mathrm{X}$ parallèle), et dans l'enregistrement des raies de diffraction (à l'aide de détecteurs à résolution en énergie, ou de détecteurs à localisation linéaire bidimensionnels, ou bien de systèmes de plaque-images à affichage digital) assurent un avenir certain et promettent une évolution enthousiasmante dans le domaine de l'analyse des contraintes par rayons X.

\section{REFERENCES BIBLIOGRAPHIQUES}

[1] Forschung mit Röntgenstrahlen, Edit. H.W. Heuck, E. Macherauch, Springer, Heidelberg, Berlin, 1995.

[2] B. EIGENMANN, E. MACHERAUCH, Röntgenographische Untersuchung von Spannungszuständen in Werkstoffen, Mat.-wiss. u. Werkstofftech. 26 (1995), partie 1: p. 148-160, partie Il: p. 199-216, partie Ill: 27 (1996), en impression, partie IV: envoyée.

[3] H.R. ISENBURGER, Bibliography on X-Ray Stress Analysis, St. John X-Ray Laboratory, California N.J. U.S.A., Ist edn. 1949, 2nd edn. 1953.

[4] J. HAUK, Schrifttum 1925-1975, HTM 31 (1976), p. 112-124.

[5] J. HAUK, Schrifttum über röntgenographische Spannungsermittlung ab 1975, HTM 37 (1982), p. 223-237.

[6] J. HAUK, Schrifttum über röntgenographische Spannungsermittlung 1982 bis 1985 sowie Ergänzungen 1981, HTM 42 (1987), p. 225-239.

[7] J. HAUK, Schrifttum über röntgenographische Spannungsermittlung 1986-1988 sowie Ergänzungen 1985, HTM 45 (1990), p. 373-388.

[8] J. HAUK, Schrifttum über röntgenographische Spannungsermittlung 1989, HTM 47 (1992), p. 189-195.

[9] J. HAUK, Schrifttum über rântgenographische Spannungsermittlung 1990, HTM 48 (1993), p. 133-136. 
[10] J. HAUK, Schrifttum über Spannungsermittlung mit Röntgen- und Neutronenstrahlen, HTM 49 (1994), p. 406-419.

[11] A.F. JOFFE, M.V. KIRPITCHEVA, Röntgenograms of Strained Crystals, Philosophical Magazine 43 (1922), p. 204-206.

[12] W. SOLLER, A New Precision X-Ray Spectrorneter, Phys.Rev. 24 (1924), p. 158-167.

[13] U. DEHLINGER, Über die Verbreiterung der Debyelinien bei kaltbearbeiteten Metallen, Z. Kristallogr. 65 (1927), p. 615-631.

[14] G.I. AKSENOW, Measurement of Elastic Stress in a Fine-Grained Material, Z. angew. Phys. USSR 6 (1929), p. 3-16.

[15] G. SACHS, J. WEERTS, Elastizitätsmessungen mit Röntgenstrahlen, Z. tech. Phys. 64 (1930), p. 344-358.

[16] F. WEVER, H. MOLLER, Uber ein Verfahren zum Nachweis innerer Spannungen, Arch. Elsenhüttenwes. 5 (1931), p. 215-218.

[17] F. WEVER, H. MOLLER, X-Ray Diffraction Patterns Showing Stress, Weld. Eng. 17 (1932), p. 35-37.

[18] F. WEVER, H. MOLLER, Uber die Präzisionsbestimmung von Gitterkonstanten mit dem Rückstrahiverfahren, Mitt. KWI Elsenforsch. Düsseidorf 15 (1933), p. 59-69.

[19] R. GLOCKER, E. OSSWALD, Einzelbestimmungen der elastischen Hauptspannungen mit Röntgenstrahlen, Z. tech. Phys. 16 (1935), p. 237-242.

[20] R. GLOCKER, B. HESS, 0. SCHAABER, Einzelbestimmungen von elastischen Spannungen mit Röntgenstrahlen 111, Z. Tech. Phys. 19 (1938), p. 194-204.

[21] W. ROMBERG, X - Ray Determination of Stress Tensor, Tech. Phys. USSR 4 (1937), p. 524-532.

[22] H. MÖLLER, J. BARBERS, Röntgenographische Untersuchung über Spannungsverteilung und Uberspannungen in Fluostahl, Mitt. KWI Elsenforsch. Düsseldorf 17 (1935), p. 157-166.

[23] VOIGT, Lehrbuch der Kristallphysik, Teubner, Berlin, 1928.

[24] A. REUSS, Berechnung der Fliessgrenze von Mischkristalien aufgrund der Plastizitätsbedingung für Einkristalle, Z. für angew. Math. und Mech. 9 (1929), p. 49-58.

[25] R. GLOCKER, Einflug einer elastischen Anisotopie auf die röntgenographische Messung von Spannungen, Z. Tech. Phys. 19 (1938), p. 289-293.

[26] E. SCHIEBOLD, Beitrag zur Theorie der Messungen elastischer Spannungen in Werkstogen mit Hilfe von RöntgenstrahlenInterferenzen, Berg- u. Hüttenm. Monatsh. 68 (1938), p. 278-295.

[27] H. MÖLLER, H. NEERFELD, Zur Berechnung röntgenographischer Elastizitätskonstanten, Mitt. KWI Eisenforsch. Düsseldorf 23 (1941), p. 97-104.

[28] E. HEYN, Eine Theorie der Verfestigung von metallischen Werkstoffen infolge Kaltreckens, Festschrift der Kaiser-Wilhelm-Gesellschaft, Springer, Berlin, 1921, p. 121-131. 
[29] G. MASING, Eigenspannungen in kaltgereckten Metallen, Z. Tech. Phys. 6 (1925), p. 569-573.

[30] J.T. NORTON, On X-Ray Determination of Stresses in Welds, Weld. J. 11 (1932) 9, p. 5-7.

[31] J.T. NORTON, X - Ray Methods of Studying Stress Relief in Welds, Weld. J. Res. Suppl. 16 (1937), p. 19-22.

[32] G. WASSERMANN, Ober Abschreckspannungen. Röntgenographische Untersuchungen zur Frage der Gitterkonstantenunterschiede, Mitt. KWI Elsenforsch. Düsseldorf 17 (1935), p. 167-174.

[33] K. NISHIHARA, K. KojIMA, X - Ray Stress Analysis of Cast Iron, Trans. Japan. SME 4 (1938), p. 110-112.

[34] R. GLOCKER, G. KEMMNITZ, A. SCHAAL, Röntgenographische Spannungsmessung bei dynamischer Beanspruchung, Arch. Elsenhüttenwes. 13 (1939/49), p. 89-92.

[35] H. KRÄCHTER, Röntgenographische Bestimmung der Formziffer gekerbter Rundstäbe, Z. Metallkde. 31 (1939), S. 114-115.

[36] ALTSCHULER, SPERANSKI, Residual Stresses caused by Grinding, Vest. Met. USSR 1 (1940), p. 15.

[37] E.M. MAHLA, Stresses in Electrodeposited Copper, Trans. Electrochem. Soc. 77 (1940), p. 145-150.

[38] E.W. MILBURN, X-Ray Diffraction Applied to Shot Peened Surfaces, Met. Treat. 12 (1945), p. 259-260.

[39] F. BOLLENRATH, V. HAUK, Röntgenspannungsmessungen an Stählen mit verschiedenem Kohlenstoffgehalt und unterschiedlicher Gefügeausbildung, $Z$. Metallkde. 37 (1946), p. 161-167.

[40] J.T. NORTON, D. ROSENTHAL, S.B. MALOOF, X-Ray Diffraction Study of the Effect of Residual Compression on the Fatigue of Notched Specimens, Weld. Res. Counc. (1946), p. 729-735.

[41] E. OSSWALD, Der Einflug einer tiefenabhängigen Spannungsverteilung auf die röntgenographische Spannungsmessung, Z. Metallkde. 39 (1948), p. 279-288.

[42] G. FROHNMEYER, E.-G. HOFMANN, Röntgenographische Spannungsmessungen an einzelnen Kristalliten eines auf Zug beanspruchten Stahls, Z. Metallkde. 43 (1952), p. 151-158.

[43] A. DURER, Verfahren zur Bestimmung der Gitterkonstanten spannungsbehafteter Proben, Z. Metallkde. 37 (1946), p. 60-62.

[44] R. GLOCKER, Bestimmung der Spannung und des Wertes der Gitterkonstanten für den spannungsfreien Zustand aus einer Röntgenruckstrahlaufnahme, Z. Metallkde. 42 (1951), p. 122-124.

[45] F. BINDER, E. MACHERAUCH, Die dehnungsfreien Richtungen des ebenen Spannungszustandes und ihre Bedeutung für röntgenographische Spannungsmessungen und Untersuchungen von Strukturen, Arch.Eisenhüttenwes. 26 (1955), p. 355-358. 
[46] H. MÖLLER, H. NEERFELD, Die Veirwendung des Interferenzzählrohrs zur röntgenographischen Spannungsmessung, Arch. Eisenhüttenwes. 19 (1948), p. 187190.

[47] S.R. MALOOF, H.R. ERARD, A Critical Evaluation of the Norelco High Angle X Ray Spectrometer for Elastic Strain Measurements, Rev. Sci. Instr. 23 (1952), p. 687-692.

[48] A.L. CHRISTENSON, E.S. ROWLAND, Residual Stress Hardened in High Carbon Steel, ASM-Preprint 1952/53.

[49] V. HAUK, Zum gegenwärtigen Stand der Spannungsmessung mit Röntgenstrahlen, Arch. Elsenhüttenwes. 26 (1955), p. 275-278.

[50] H. MÖLLER, F. BRASSE, Ausmessung von Röntgen-Interferenzkurven mit einem Szintillationszähler, Arch. Eisenhüttenwes. 28 (1957), p. 831-837.

[51] G.B. GREENOUGH, Residual Lattice Strains in Plastically Deformed Polycrystalline Metal Aggergates, Proc. Roy. Soc. London A 197 (1949), p. 556567.

[52] E. MACHERAUCH, Die Eigenspannungsbestimmung mit Röntgenstrahlen, 111. Internat. Kolloquium der Hochschule für Elektrotechnik 11 menau (1958), p. 359-364.

[53] E. MACHERAUCH, P. MÜLLER, Das $\sin ^{2} \psi$-Verfahren der röntgenographischen Spannungsmessung, $Z$. angew. Physik 13 (1961), p. 305-312.

[54] E. MACHERAUCH, P. MÜLLER, Ermittlung der röntgenographischen Werte der elastischen Konstanten von kalt gerecktem Armco-Eisen und Chrom-MolybdänStahl, Arch. Eisenhüttenw. 29 (1958), p. 257-261.

[55] U. WOLFSTIEG, Röntgenographische Spannungsmessungen mit breiten Linien, Arch. Elsenhüttenwes. 30 (1959), p. 447-450.

[56] S. KARASHIMA, Neue Fortschritte bei röntgenographischen Spannungsmessungen in Japan, HTM 20 (1965), p. 131-135.

[57] H. NEFF, Mobiles Röntgendiffraktometer, Siemens Bericht 1963.

[58] K. KOLB, E. MACHERAUCH, Ein Röntgenstrahlgoniometer nach dem BraggBrentano Prinzip zur röntgenographischen Spannungsmessung, J. Soc. Mater. Sci. Japan 13 (1964), p. 918-919.

[59] F. BOLLENRATH, V. HAUK, E.H. MÜLLER, Zur Berechnung der vielkristallinen Elastizitätskonstanten aus den Werten der Einkristalle, Z. Metallkde. 58 (1967), p. 76-82.

[60] G. FANINGER, Die elastischen Konstanten von Kupfer-Nickel Vielkristalien, Z. Metallkde. 60 (1969), p. 601-605.

[61] T. SHIRAIWA, Y. SAIKAMOTO, The Effect of X-Ray Penetration on the Stress Measurements of Hardened Steel, J. Soc. Mat. Sci. Japan 16 (1967), p. 943-947.

[62] S. TAIRA, K. HAYASHI, Y. NISHINO, X-Ray Investigation on the Strength of Inhomogeneous Metallic Materials (Deformation Behaviour and X - Ray Elastic Constants of Cast Iron), J. Soc. Mat. Sci. Japan 16 (1967), p. 1020-1026. 
[63] S. TAIRA, K. HAYASHI, Z. WATASE, X-Ray Investigation on the Deformation of Polykrystalline Metais (On the Change in X-Ray Elastic Constants by Plastic Deformation), J. Soc. Mat. Sci. Japan 17 (1968), p. 1151-1157.

[64] C. KELLY, E. EICHEN, Computer Controlled X-Ray Diffraction Measurement of Residual Stress, Adv. X-Ray Analysis 16 (1972), p. 344-353.

[65] M.YA. FUKS, L.I. GLADKIKH, On some Distinctive Features of the X - Ray Method of Measuring Elastic Stresses, Zavodskaya Laboratoriya 31 (1965), p. 978-982.

[66] A. DIAMENT, La méthode radiocristallographique d'analyse de contraintes, ses possibilités et ses limites, Rev. Franc. Mec. 39 (1974), P55-62.

[67] H. WALBURGER, FachausschuBsitzung Spannungsmegtechnik, Plättig (1973).

G. FANINGER, H. WALBURGER, Anomalien bei der röntgenographischen Ermittlung von Schleifeigenspannungen, HTM 31 (1976), p. 79-82.

[68] P.D. EVENSCHOR, V. HAUK, Uber nichtlineare Netzebenenabstandsverteilungen bei röntgenographischen Dehnungsmessungen, Z. Metallkde. 66 (1975), p. 167-168.

[69] E. CHRIST, H. KRAUSE, Ober die Auswertung nichtlinearer d. vs.sin ${ }^{2} \psi$ Verteilungen, Z. Metallkde. 66 (1975), S. 615-618.

[70] H. DÖLLE, V. HAUK, Röntgenographische Spannungsermittlung für Eigenspannungssysteme allgemeiner Orientierung, HTM 31 (1976), p. 165-168.

[71] M. WAKABAYASHI, N. NAKAYAMA, A. NAGATA, Influence of Grinding Direction on Residual Strains Measured by X-ray, J. of the Jap. Soc. Prec. Eng. 43 (1977), p. 661-667.

[72] C.S. BARRETT, P. PREDECKI, Stress Measurement in Polymeric Materials by XRay Diffraction, Polymer Engineering and Science 16 (1976), p. 602-608.

[73] M. NAGAO, S. KUSUMOTO, Polychromatic X-Ray Stress Analysis and its Application, in: Nondestructive Evaluation of Materials, édit. J.J. Burke, V. Weis, Plenum Press, New York, London 1979, p. 221-256.

[74] M.R. JAMES, J.B. COHEN, The Application of a Position Sensitive X - Ray Detector to the Measurement of Residual Stresses, Adv. X-Ray Anal. 19 (1976), p. 695-708.

[75] M. BARRAL, J.M. SPRAUEL, J.L. LEBRUN, G. MAEDER, S. MEGTERT, On the Use of Synchrotron Radiation for the Study of the Mechanical Behaviour of Materials, Adv. X-Ray Anal. 27 (1984), p. 149-158.

[76] H.A. CROSTACK, W. REIMERS, G. ECKOLD, Analysis of Residual Stresses in Single Grains of Polycrystalline Materials, in: Residual Stresses in Science and Technology, édit. E. Macherauch, V. Hauk, DGM Informationsgeselischaft $\mathrm{mbH}$, Oberursel 1987, vol. 1, p. 281-288.

[77] V. HAUK, W.K. KRUG, R.W.M. OUDELHOVEN, L. PINTSCHOVIUS, Calculation of Lattice Strains in Crystallites with an Orientation Corresponding to the Ideal Rolling Texture of Iron, Z. Metallkde. 79 (1988), p. 159-163.

[78] W. LODE, A. PEITER, Grundsätzliche Erweiterungsmöglichkeiten der RöntgenVerformungsmesstechnik, Metall 35 (1981), p. 758-762. 
[79] B. EIGENMANN, B. SCHOLTES, E. MACHERAUCH, Röntgenographische Eigenspannungsmessungen an texturbehafteten PVD-Schichten aus Titankarbid, HTM 43 (1988), p. 208-211.

[80] S. TORBATY, J.M. SPRAUEL, G. MAEDER, P.H. MARKHO, On the X-Ray Diffraction Method of Measurement of Triaxial Stresses with Particular Reference to the Angle 2 $\theta_{0}$, Advances in X-Ray Anal. 26 (1983), p. 245-253.

[81] M. BARRAL, J.M. SPRAUEL, G. MAEDER, Stress Measurement on Textured Material Characterised by its Orientation Distribution Fonction, dans: Eigenspannungen, édit. E. Macherauch, V. Hauk, DGM Informationsgesellschaft mbH, Oberursel, 1983, vol. 2, p. 31-47.

[82] M. BARRAL, J.L. LEBRUN, J.M. SPRAUEL, G. MAEDER, X-Ray Macrostress Determination on Textured Material. Use of the ODF for Calculating the $X$ - Ray Compliances, Metall. Trans. 18 A (1987), p.1229-1238.

[83] W. SERRUYS, P. VAN HOUTTE, E. AERNOUT, X- Ray Measurement of Residual Stresses in Textured Materials with the Aid of Orientation Distribution Fonctions, dans: Residual Stresses in Science and Technology, édit. E. Macherauch, V. Hauk, DGM Informationsgeseilschaft mbH, Oberursel 1987, vol. 1, p. 417-424.

[84] V. HAUK, A. TROOST, D. LEY, Evaluation of (Residual) Stresses in Semicristalline Polymers by X - Rays, Adv. Polymer Tech 7 (1987), p. 389-396.

[85] L. DE BUYSER, P. VAN HOUTTE, E. AERNOUDT, $\mathbf{X}$ - Ray Measurement of Residual Stress in Thin Layers, Residual Stresses - Measurement, Calculation, Evaluation, édit. V. Hauk, H. Hougardy, E. Macherauch, DGMInformationsgeseilschaft mbH, Oberursel, 1991, p. 27-32.

[86] H. RUPPERSBERG, I. DETEMPLE, J. KRIER, Evaluation of Strongly Non-Linear Surface-Stress Fields $\sigma_{x x}(z)$ and $\sigma_{y y}(z)$ from Diffraction Experiments, Phys. Stat. Sol. 116 (1989), p. 681-689.

[87] B. EIGENMANN, E. MACHERAUCH, Determination of Grinding Residual Stress States in Surface Layers of Engineering Ceramics Using Synchrotron $X$ - Rays, $Z$. Metallkde. 86 (1995), p. 84-90.

[88] B. EIGENMANN, B. SCHOLTES, E. MACHERAUCH, An Improved Technique for X-Ray Residual Stress Determinations on Ceramics with Steep Subsurface Stress Gradients, Residual Stresses-111, édit. H. Fujiwara, T. Abe, K. Tanaka, Elsevier Applied Science, London, New York, 1992, vol. 1, p. 601-606.

[89] T. LEVERENZ, B. EIGENMANN, E. MACHERAUCH, Das Abschnitt-PolynomVerfahren zur zerstörungsfreien Ermittlung gradienten-behafteter Eigenspannungszustände in den Randschichten von bearbeiteten Keramiken, envoyé chez $Z$. Metallkde. 